\title{
TITLE:
}

\section{Analysis and flamelet modelling for spray combustion}

AUTHOR(S):

Baba, Yuya; Kurose, Ryoichi

CITATION:

Baba, Yuya ...[et al]. Analysis and flamelet modelling for spray

combustion. Journal of Fluid Mechanics 2008, 612: 45-79

ISSUE DATE:

2008-10-10

URL:

http://hdl.handle.net/2433/88947

RIGHT:

Copyright (c) Cambridge University Press 2008 


\title{
Analysis and flamelet modelling for spray combustion
}

\author{
YUYA BABA ${ }^{1}$ AND R YOICHI KUROSE \\ ${ }^{1}$ Earth Simulator Center, Japan Agency for Marine-Earth Science and Technology (JAMSTEC), \\ 3173-25 Showa-machi, Kanazawa-ku Yokohama, Kanagawa 236-0001, Japan \\ babay@jamstec.go.jp \\ ${ }^{2}$ Department of Mechanical Engineering and Science, and Advanced Research Institute of Fluid \\ Science and Engineering, Kyoto University, Yoshida-honmachi, Sakyo-ku, Kyoto 606-8501, Japan \\ kurose@mech.kyoto-u.ac.jp
}

(Received 21 February 2007 and in revised form 19 May 2008)

The validity of a steady-flamelet model and a flamelet/progress-variable approach for gaseous and spray combustion is investigated by a two-dimensional direct numerical simulation (DNS) of gaseous and spray jet flames, and the combustion characteristics are analysed. A modified flamelet/progress-variable approach, in which total enthalpy rather than product mass fraction is chosen as a progress variable, is also examined. DNS with an Arrhenius formation, in which the chemical reaction is directly solved in the physical flow field, is performed as a reference to validate the combustion models. The results show that the diffusion flame is dominant in the gaseous diffusion jet flame, whereas diffusion and premixed flames coexist in the spray jet flame. The characteristics of the spray flame change from premixed-diffusion coexistent to diffusion-dominant downstream. Comparisons among the results from DNS with various combustion models show the modified flamelet/progress-variable approach to be superior to the other combustion models, particularly for the spray flame. Where the behaviour of the gaseous total enthalpy is strongly affected by the energy transfer (i.e. heat transfer and mass transfer) from the dispersed droplet, and this effect can be accounted for only by solving the conservation equation of the total enthalpy. However, even the DNS with the modified flamelet/progress-variable approach tends to underestimate the gaseous temperature in the central region of the spray jet flame. To increase the prediction accuracy, a combustion model for the partially premixed flame for the spray flame is necessary.

\section{Introduction}

Spray combustion is utilized in a number of engineering applications, such as energy conversion and propulsion devices. It is therefore necessary to precisely predict the combustion behaviour of the fuel spray when designing and operating equipment. However, since spray combustion is a complex phenomenon, in which the dispersion of fuel droplets, their evaporation and a chemical reaction of the fuel vapour with oxidizer take place simultaneously and interact with each other, the underlying physics governing these processes have not been well established. In order to understand the spray combustion behaviour, numerical simulations based on direct numerical simulations (DNS) or large-eddy simulations (LES) have recently been performed.

DNS is an appropriate tool for discussing the detailed spray combustion mechanism associated with the interaction between turbulence and reaction, since all scales for 
chemical reactions as well as hydrodynamics are resolved simultaneously. Réveillon \& Vervisch (2005) and Domingo, Vervisch \& Réveillon (2005) performed twodimensional DNS of spray jet flames and discussed the detailed characteristics in terms of the topology of the droplet group combustion regimes and the partially premixed model, respectively. Nakamura et al. (2005) also investigated the droplet group combustion behaviour, under various droplet-size and equivalence-ratio conditions by two-dimensional DNS of spray flames formed in laminar counterflow diffusion flames. Watanabe et al. $(2007,2008)$ extended the work of Nakamura et al. (2005) and discussed the flamelet characteristics of the spray flames. These works reveal that at present the DNS of spray flames is limited to two-dimensional simulations, since limits on computational costs make it impossible to conduct three-dimensional simulations to clarify the three-dimensional spray flame behaviour.

On the other hand, LES, in which the gaseous-phase behaviour is spatially filtered and the unresolved scales are modelled by subgrid-scale (SGS) models, is attractive, since it can be applied to three-dimensional spray combustion fields even for large-scale industrial devices. Ham et al. (2003) and Kurose et al. (2003, 2004) performed LES of an turbulent spray combustion in a realistic gas turbine and a solid/spray jet flame, respectively, and discussed the validity. These works appear to be successful, but also reveal a further question concerning the combustion model for spray flames, as follows.

In LES or RANS (Reynolds-averaged Navier-Stokes) modelling of gaseous fuel diffusion combustion, the flamelet models proposed by Peters $(1984,2000)$ have been widely used in different formulations such as the steady-flamelet model (Cook, Riley \& Kosály 1997), unsteady-flamelet model (Pitsch 2000; Pitsch \& Steiner 2000), and flamelet/progress-variable approach (Pierce \& Moin 2004). In these flamelet models, in general, the conservation equations of mass, momentum and mixture fraction $Z$ as a conserved scalar are solved in the flow field (physical space), and temperature and chemical species mass fractions at each position are identified by referring to a database, a so-called 'chemtable' (i.e. chemistry table, flamelet library). The chemtable is obtained by solving a one-dimensional flamelet equation in the $Z$ space, and $Z$ and the scalar dissipation rate $\chi\left(=2 D_{Z} \nabla Z \cdot \nabla Z\right)$ are used as parameters to relate the physical space to the $Z$ space. Here, $D_{Z}$ is the diffusion coefficient of $Z$.

For the spray combustion, on the other hand, $Z$ is no longer a conserved scalar because of the mass transfer (i.e. evaporation of fuel) between the dispersed droplet phase and the carrier gaseous phase (Watanabe et al. 2007, 2008). Consequently, the behaviour of variables such as temperature and chemical species mass fractions versus $Z$ and $\chi$ in two-phase combustion becomes complicated and cannot be identified by these parameters alone. In addition, without solving the energy equation, the heat transfer between the dispersed droplet phase and the carrier gaseous phase cannot be taken into account. The heat transfer, including the heat loss due to droplet evaporation, which is referred to as the droplet cooling effect, is found to affect the spray flame temperature (Nakamura et al. 2005). Although Hollmann \& Gutheil (1998) proposed a steady-flamelet-based model for spray flames, their modelling needs many parameters to account for the spray characteristics and lacks universality. The flamelet/progress-variable approach proposed by Pierce \& Moin (2004), which employs an additional parameter called the progress variable to capture the flame lift, local extinction and re-ignition dynamics, may also improve the numerical accuracy for spray combustion, because this approach can provide intrinsic information concerning the relation between $Z$ and the chemical reaction. In the original model, however, again the heat transfer between the phases cannot be considered, since 
a product mass fraction unrelated to thermal energy is chosen as the progress variable.

In this study, we propose a modified flamelet/progress-variable approach, in which enthalpy is employed as the progress variable (referred to as FPVA-E, hereafter), and its applicability to gaseous and spray flames is investigated by performing twodimensional DNS of gaseous and spray jet flames. The results are compared with cases where an Arrhenius formation, a steady-flamelet model and the conventional flamelet/progress-variable approach, in which the product mass fraction is chosen as the progress variable, are employed as the combustion models (referred to as ARF, SFM and FPVA-P, respectively), and the detailed spray combustion mechanism is discussed in terms of the modelling. Originally, combustion models such as SFM and FPVA were intended for use in connection with SGS models for LES or RANS of the carrier gaseous phase. However, in order to avoid discussion of the effect of the SGS contributions on numerical accuracy, a numerical method using fine resolution without the SGS models is chosen here. In this paper, we simply call this method DNS, regardless of the combustion model.

The numerical procedure, including the governing equations, combustion models, and numerical conditions, is described in $§ 2$. The detailed combustion behaviour and the validity of the combustion models are discussed for gaseous combustion and spray combustion in $\S 3$ and $\S 4$, respectively. Finally, the main findings are summarized in $\S 5$.

\section{Numerical simulations}

In this paper, we refer to the numerical method, in which non-filtered and nonaveraged equations of the carrier gaseous phase are solved, as DNS regardless of the combustion model. The combustion models compared are the Arrhenius formation (ARF), steady-flamelet model (SFM), and flamelet/progress-variable approach (FPVA). It is assumed that DNS with ARF reveals true flame behaviour.

\subsection{Governing equations for DNS with Arrhenius formation ( $A R F$ )}

The conservation equations of the mass, momentum, internal energy and mass fractions of chemical species are solved to describe the carrier gaseous phase behaviour. The set of governing equations based on a low-Mach-number formulation is (Nakamura et al. 2005)

$$
\begin{gathered}
\frac{\partial \rho}{\partial t}+\frac{\partial \rho u_{j}}{\partial x_{j}}=S_{\rho}, \\
\frac{\partial \rho u_{i}}{\partial t}+\frac{\partial \rho u_{j} u_{i}}{\partial x_{j}}=-\frac{\partial p}{\partial x_{i}}+\frac{\partial \sigma_{i j}}{\partial x_{j}}+S_{u_{i}}, \\
\frac{\partial \rho h}{\partial t}+\frac{\partial \rho u_{j} h}{\partial x_{j}}=\frac{\partial}{\partial x_{j}}\left(\rho a \frac{\partial h}{\partial x_{j}}\right)+S_{h}, \\
\frac{\partial \rho Y_{i}}{\partial t}+\frac{\partial \rho u_{j} Y_{i}}{\partial x_{j}}=\frac{\partial}{\partial x_{j}}\left(\rho D_{i} \frac{\partial Y_{i}}{\partial x_{j}}\right)+\dot{m}_{i}+S_{Y_{i}},
\end{gathered}
$$

where $\rho, u_{i}, h, Y_{i}$ are the density, velocity, total enthalpy and mass fraction of the $i$ th species, respectively; $\dot{m}_{i}$ is the mass production or consumption rate of the $i$ th species, as shown later; $\sigma_{i j}$ is the viscous stress tensor given by

$$
\sigma_{i j}=\mu\left(\frac{\partial u_{i}}{\partial x_{j}}+\frac{\partial u_{j}}{\partial x_{i}}\right)-\frac{2}{3} \mu \delta_{i j} \frac{\partial u_{k}}{\partial x_{k}},
$$




\section{Y. Baba and R. Kurose}

where $\mu$ is the viscosity. In (2.3), the diffusion coefficient of the enthalpy $a$ is given by $\rho a=\lambda / c_{p}$. In (2.4), the unity Lewis number assumption, in which all diffusion coefficients of species are given by $\rho D_{i}=\lambda / c_{p}$, is introduced.

The phase coupling is implemented using a Eulerian/Lagrangian method. $S_{\rho}, S_{u_{i}}$, $S_{h}$ and $S_{Y_{i}}$ are the phase coupling terms between the carrier gaseous phase and the dispersed droplet phase given as

$$
\begin{aligned}
S_{\rho} & =-\frac{1}{\Delta V} \sum_{N} \frac{\mathrm{d} m_{d}}{\mathrm{~d} t}, \\
S_{u_{i}} & =-\frac{1}{\Delta V} \sum_{N} \frac{\mathrm{d}}{\mathrm{d} t}\left(m_{d} v_{i}\right), \\
S_{h} & =-\frac{1}{\Delta V} \sum_{N}\left[\frac{1}{2} \frac{\mathrm{d}}{\mathrm{d} t}\left(m_{d} v_{i} v_{i}\right)+\frac{\mathrm{d}}{\mathrm{d} t}\left(c_{L} m_{d} T_{d}\right)\right], \\
S_{Y_{i}} & =-\frac{1}{\Delta V} \sum_{N} \frac{\mathrm{d} m_{d}}{\mathrm{~d} t} \quad \text { for } Y_{i}=Y_{V},
\end{aligned}
$$

where $c_{L}$ is the heat capacity of the droplet, $\Delta V$ the control volume and $Y_{V}$ the mass fraction of fuel vapour. Summation $\sum_{N}$ denotes the contributions of sources surrounding the liquid droplets, from the dispersed phase to the carrier gaseous phase. Linear interpolation and extrapolation with weighting factors are used for the phase coupling (Miller \& Bellan 1999). Other variables will be explained later.

Individual droplets are tracked in a Lagrangian framework under the assumption that the droplet volume is negligible. The governing equations for the droplet position $X_{i}$, velocity $v_{i}$, temperature $T_{d}$ and mass $m_{d}$ are given by (Miller, Harstad \& Bellan 1998)

$$
\begin{gathered}
\frac{\mathrm{d} X_{i}}{\mathrm{~d} t}=v_{i}, \\
\frac{\mathrm{d} v_{i}}{\mathrm{~d} t}=\frac{F_{i}}{m_{d}}=\left(\frac{f_{1}}{\tau_{d}}\right)\left(u_{i}-v_{i}\right), \\
\frac{\mathrm{d} T_{d}}{\mathrm{~d} t}=\frac{Q_{d}+\dot{m}_{d} L_{V}}{m_{d} c_{L}}=\frac{N u}{3 P r}\left(\frac{f_{2}}{\tau_{d}}\right)\left(\frac{c_{p}}{c_{L}}\right)\left(T-T_{d}\right)+\frac{\dot{m}_{d}}{m_{d}} \frac{L_{V}}{c_{L}}, \\
\frac{\mathrm{d} m_{d}}{\mathrm{~d} t}=\dot{m}_{d}=-\frac{m_{d}}{\tau_{d}}\left(\frac{S h}{3 S c}\right) \ln \left(1+B_{M}\right),
\end{gathered}
$$

where $F_{i}$ is the drag force, $Q_{d}$ is the heat flux, $L_{V}$ is the latent heat of droplet evaporation, given as a function of the droplet temperature and $\mathrm{Nu}, \mathrm{Pr}, \mathrm{Sh}$ and $\mathrm{Sc}$ are the Nusselt, Prandtl, Sherwood and Schmidt number, defined below.

The particle response time of the droplet is $\tau_{d}$ defined by

$$
\tau_{d}=\frac{\rho_{d} d_{d}^{2}}{18 \mu},
$$

where $\rho_{d}$ and $d_{d}$ are the droplet density and diameter, respectively. The internal droplet temperature and density are assumed to be uniform. In addition, the lift force due to fluid shear and breakup and collision of the droplets are neglected (Kurose \& Komori 1999; Apte, Gorokhovski \& Moin 2003). As will be shown later, droplets become dense close to the injector and, in this area, spray dispersion is not realistic. 
However, because the combustion phenomena far from the injector are focused on in this study, these assumptions are considered to be acceptable.

The mass transfer number $B_{M}$ is calculated from the mass fraction of the fuel in the carrier gaseous phase $Y_{V}$ and that at the droplet's surface $Y_{V, s}$ as

$$
B_{M}=\left(Y_{V, s}-Y_{V}\right) /\left(1-Y_{V}\right)
$$

$Y_{V, s}$ is evaluated using the Clausius-Clapeyron relation under the saturation pressure condition as

$$
\begin{gathered}
Y_{V, s}=\frac{X_{V, s}}{X_{V, s}+\left(1-X_{V, s}\right) \bar{W} / W_{V}}, \\
X_{V, s}=\frac{P_{a t m}}{P} \exp \left[\frac{L_{V}}{R_{V}}\left(\frac{1}{T_{B L}}-\frac{1}{T_{d}}\right)\right]-\left(\frac{2 L_{k}}{d_{d}}\right) \beta,
\end{gathered}
$$

where $X_{V, s}$ is the mole fraction of fuel vapour at the droplet surface, $P_{a t m}$ is the atmospheric pressure, $P$ is the pressure of the carrier gaseous phase, $R_{V}$ is the gas constant for fuel vapour, and $T_{B L}$ is the boiling temperature of the liquid droplet. $\bar{W}$ and $W_{V}$ are the averaged molecular weights of the carrier gaseous phase and the molecular weight of the fuel vapour, respectively. In (2.16), a non-equilibrium effect is considered using the Langmuir-Knudsen evaporation law (Bellan \& Summerfield $1978) ; \beta$ is the non-dimensional evaporation parameter given by

$$
\beta=-\left(\frac{\rho_{d} P r}{8 \mu}\right) \frac{\mathrm{d} d_{d}^{2}}{\mathrm{~d} t} .
$$

The value of the Knudsen layer thickness $L_{k}$ is computed by

$$
L_{k}=\frac{\mu\left[2 \pi T_{d}\left(R / W_{V}\right)\right]^{1 / 2}}{S c P},
$$

where $R$ is the universal gas constant. The Nusselt and Sherwood numbers are given based on the Ranz-Marshall correlation as

$$
N u=2+0.552 \operatorname{Re}_{s l}^{1 / 2} \operatorname{Pr}^{1 / 3}, \quad S h=2+0.552 R e_{s l}^{1 / 2} S c^{1 / 3} .
$$

The modified correlation for the drag force, the reduction effect due to including blowing velocity, is (Kurose et al. 2003)

$$
\begin{gathered}
f_{1}=\frac{1+0.0545 R e_{s l}+0.1 R e_{s l}^{1 / 2}\left(1-0.03 R e_{s l}\right)}{1+b\left|R e_{b}\right|^{c}}, \\
b=0.06+0.077 \exp \left(-0.4 R e_{s l}\right), \quad c=0.4+0.77 \exp \left(-0.04 R e_{s l}\right),
\end{gathered}
$$

where $R e_{s l}=\rho u_{s l} d_{d} / \mu$ and $R e_{b}=\rho u_{b} d_{d} / \mu$ are the particle Reynolds numbers based on the slip velocity $u_{s l}=\left|u_{i}-v_{i}\right|$ and the blowing velocity $u_{b}$, respectively. On the other hand, $f_{2}$ is the correction to the heat transfer given by $f_{2}=\beta /\left(e^{\beta}-1\right)$.

A one-step global reaction of n-decane oxidation,

$$
\mathrm{C}_{10} \mathrm{H}_{22}+31 / 2 \mathrm{O}_{2} \rightarrow 10 \mathrm{CO}_{2}+11 \mathrm{H}_{2} \mathrm{O}
$$

is considered here to reduce the computational cost. In ARF, the mass consumption rate of the evaporated fuel (n-decane) $\dot{m}_{V}$ is given by (Westbrook \& Dryer 1984)

$$
\dot{m}_{V}=W_{V} A T^{n}\left(\frac{\rho Y_{V}}{W_{V}}\right)^{a}\left(\frac{\rho Y_{O}}{W_{O}}\right)^{b} \exp \left(-\frac{E}{R T}\right) .
$$


Here, $A$ is the pre-exponential factor, $E$ the activation energy, and $n, a$ and $b$ the coefficients for hydrocarbon fuels. $W_{O}$ is the molecular weight of the oxidizer and $Y_{V}, Y_{O}$ are the mass fractions of the fuel and oxidizer, respectively. For the n-decane oxidation reaction, those constants are $A=3.8 \times 10^{11}, E=30 \mathrm{kcal}, n=0, a=0.25$ and $b=1.5$. The chemical species considered are $\mathrm{C}_{10} \mathrm{H}_{22}, \mathrm{CO}_{2}, \mathrm{H}_{2} \mathrm{O}, \mathrm{O}_{2}$ and $\mathrm{N}_{2}$, and their production or consumption rates $\dot{m}_{i}$ in (2.4) are calculated by using the value of $\dot{m}_{V}$ and the equivalence relation. The thermodynamics and transport properties such as viscosity and thermal conductivity are calculated using the CHEMKIN database (Kee et al. 1986; Kee, Rupley \& Miller 1989).

\subsection{Steady-flamelet model (SFM)}

In SFM, the conservation equations of mass (2.1), momentum (2.2), and the transport equation of the mixture fraction $Z$ are solved for the carrier gaseous phase in the physical space, and temperature and chemical species mass fractions at each position are obtained by referring to a chemtable. In general, the chemtable is generated beforehand by solving a one-dimensional flamelet equation in the $Z$ space and tabulating variables such as the temperature and chemical species mass fractions, with respect to $Z$ and the scalar dissipation rate

$$
\chi=2 D_{Z} \frac{\partial Z}{\partial x_{j}} \frac{\partial Z}{\partial x_{j}},
$$

where $D_{Z}$ is the diffusion coefficient of $Z$, which is given by $\rho D_{Z}=\lambda / c_{p}$ under the unity Lewis number assumption. It is considered that $\chi$ characterizes the strain rate of the local flame structure.

The transport equation of $Z$ is

$$
\frac{\partial \rho Z}{\partial t}+\frac{\partial \rho u_{j} Z}{\partial x_{j}}=\frac{\partial}{\partial x_{j}}\left(\rho D_{Z} \frac{\partial Z}{\partial x_{j}}\right)+S_{Z} .
$$

Here, the phase coupling term with evaporating droplets, $S_{Z}$, is required only for the spray combustion, not for the gaseous combustion. This is derived as follows. The definition of $Z$ is

$$
Z=\frac{\phi Y_{V} / Y_{V, 0}-Y_{O} / Y_{O, 0}+1}{\phi+1},
$$

where $Y_{V, 0}$ and $Y_{O, 0}$ are the mass fractions of fuel vapour and oxidizer in $Z$ space (i.e. $Y_{V, 0}=1.0$ and $\left.Y_{O, 0}=0.2324\right)$, respectively; $\phi$ is the chemical equivalence ratio defined as $\phi=\left(v_{O} W_{O} Y_{V, 0}\right) /\left(v_{V} W_{V} Y_{O, 0}\right)$, where $v_{O}$ and $v_{V}$ are the stoichiometric coefficients of oxidizer and fuel of the n-decane oxidation reaction, respectively. The source term of $Z$ in (2.25), $S_{Z}$ is derived in conservative form using relation (2.26) as

$$
\frac{\partial \rho Z}{\partial t}=\frac{1}{\phi+1}\left(\frac{\phi}{Y_{V, 0}} \frac{\partial \rho Y_{V}}{\partial t}-\frac{1}{Y_{O, 0}} \frac{\partial \rho Y_{O}}{\partial t}+\frac{\partial \rho}{\partial t}\right) .
$$

Here, the time variations of $Y_{V}$ and $Y_{O}$ due to the droplet evaporation are

$$
\frac{\partial \rho Y_{V}}{\partial t}=S_{Y_{V}}, \quad \frac{\partial \rho Y_{O}}{\partial t}=0 .
$$

This leads to $S_{Z}$ being

$$
S_{Z}=\frac{\partial \rho Z}{\partial t}=\frac{1}{\phi+1}\left(\phi S_{Y_{V}}-0+S_{Y_{V}}\right)=S_{Y_{V}} .
$$


Here, the relation of the time variation of $\rho$ due to the droplet evaporation, $\partial \rho / \partial t=$ $S_{\rho}=S_{Y_{V}}$, is used.

In SFM, variables such as the gaseous temperature and chemical species mass fractions are obtained from the chemtable using the two parameters $Z$ and $\chi$, as described earlier. Hence, these variables can be expressed as functions of $Z$ and $\chi$, i.e.

$$
T=T(Z, \chi), \quad Y_{i}=Y_{i}(Z, \chi) .
$$

\subsection{Flamelet/progress-variable approach (FPVA)}

In FPVA, the conservation equations of mass (2.1), momentum (2.2), and the transport equations of $Z$ (2.25) and one additional tracking scalar called the progress variable $C$ are solved. The progress variable $C$ must be non-conserved, independent of $Z$, and characterize $\chi$ as

$$
\chi=\chi(Z, C) .
$$

Consequently, the variables in reacting flows are represented by the reference parameters of $Z$ and $C$, rather than $Z$ and $\chi$ in SFM. This can be expressed as

$$
T=T(Z, C), \quad Y_{i}=Y_{i}(Z, C) .
$$

It should be noted here that the chemtables used for SFM and FPVA are essentially identical.

In the conventional FPVA proposed by Pierce \& Moin (2004), the product mass production is chosen as $C\left(=Y_{\mathrm{CO} 2}+Y_{\mathrm{H} 2 \mathrm{O}}\right)$ (referred to as FPVA-P). The transport equation is

$$
\frac{\partial \rho C}{\partial t}+\frac{\partial \rho u_{j} C}{\partial x_{j}}=\frac{\partial}{\partial x_{j}}\left(\rho D_{C} \frac{\partial C}{\partial x_{j}}\right)+\rho \dot{\omega}_{C},
$$

where $D_{C}$ is the diffusion coefficient of $C$, which is given by $\rho D_{C}=\lambda / c_{p}$ under the unity Lewis number assumption; $\dot{\omega}_{C}$ is the production rate of $C$. The definition of $C$ leads $\dot{\omega}_{C}$ to be a sum of the mass production rates of combustion products as

$$
\rho \dot{\omega}_{C}=\dot{m}_{C O 2}+\dot{m}_{H 2 O} .
$$

This value is obtained from the chemtable. Pierce \& Moin state that this FPVA-P is superior to SFM in capturing and reproducing flame lift, local extinction and reignition dynamics, because it provides intrinsic information about chemical reaction. However, the heat transfer between the dispersed droplet phase and carrier gaseous phase cannot be taken into account. Therefore, we propose a modified FPVA using total enthalpy $h=\sum_{i=1}^{N} h_{i} Y_{i}$ as $C$ (referred to as FPVA-E).

In gaseous combustion, the total enthalpy generally cannot be the progress variable. This is because the behaviour of the total enthalpy is very similar to that of the mixture fraction. As discussed later, however, the total enthalpy can be the progress variable when there exists a specific boundary condition for the total enthalpy or the total enthalpy is not a conserved scalar such that the last term (source term) on the righthand side of (2.3) is non-zero. These conditions appear when high-temperature coflow for the flame stabilization, heat loss from walls (Pitsch \& Duchamp de Lageneste 2002), radiation and so on are considered. In spray combustion, on the other hand, the total enthalpy can be the progress variable in spite of the above conditions, since it varies as the reaction proceeds through fuel evaporation. This is evident from (2.3), in which the last term (source term) on the right-hand side could be non-zero, as written below again. 


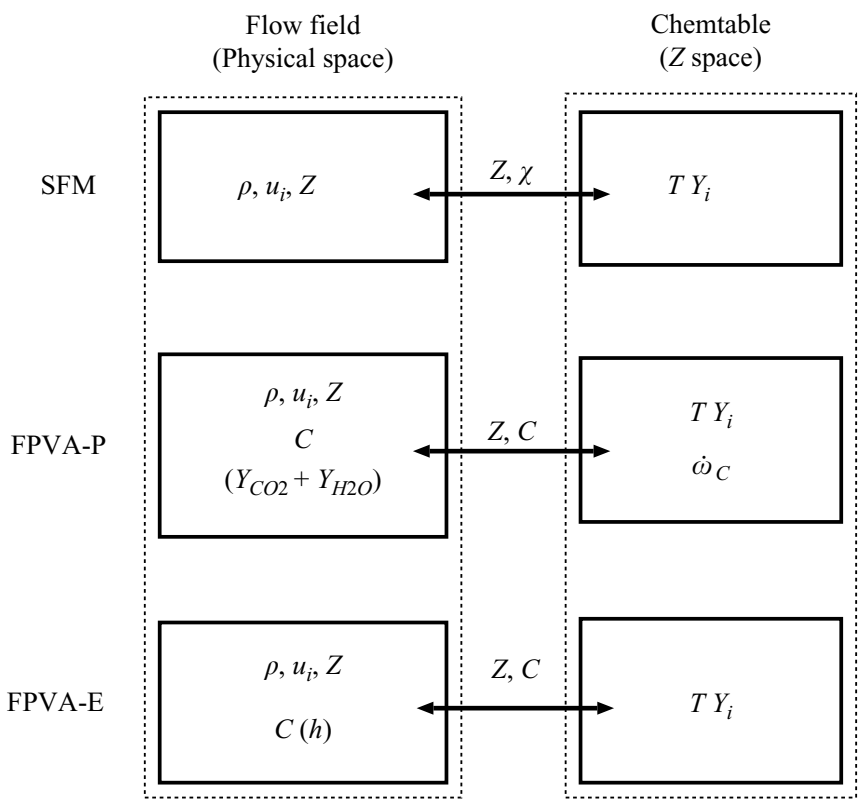

FIGURE 1. Schematic diagram of data interaction between physical space and $Z$ space.

The transport equation for $C$, i.e. $h$, is given by

$$
\frac{\partial \rho C}{\partial t}+\frac{\partial \rho u_{j} C}{\partial x_{j}}=\frac{\partial}{\partial x_{j}}\left(\rho D_{C} \frac{\partial C}{\partial x_{j}}\right)+S_{C},
$$

where $S_{C}$ is the phase coupling term corresponding to that in the conservation equation of energy for ARF (2.8):

$$
S_{C}=-\frac{1}{\Delta V} \sum_{N}\left[\frac{1}{2} \frac{\mathrm{d}}{\mathrm{d} t}\left(m_{d} v_{i} v_{i}\right)+\frac{\mathrm{d}}{\mathrm{d} t}\left(c_{L} m_{d} T_{d}\right)\right] .
$$

\subsection{Generation of the chemtable}

In the employment of SFM or FPVA, a chemtable is needed. Figure 1 shows a schematic diagram of the flamelet concept in terms of the data interaction between the flow field in the physical space (the two-dimensional DNS space, in this study) and the chemtable in the $Z$ space. In FPVA, the value of $\chi$ is represented by $C$. It should be noted that combustion models such as SFM and FPVA should be generally used in connection with SGS models for LES or RANS of the flow field, and therefore for LES and RANS, the filtered reference variables $Z, \chi$ and $C$, and all filtered variables in physical space should relate to non-filtered variables in the chemtable by probability density funtions (PDFs) (e.g. steady-flamelet, Cook et al. 1997, unsteady-flamelet, Pitsch 2000; Pitsch \& Steiner 2000).

The chemtable is generated by conducting one-dimensional counterflow flame calculations for various strain rates and tabulating the set of variables data obtained with respect to $Z$ and $\chi$. Under the unity Lewis number assumption, the onedimensional flamelet equation converted into the $Z$ space becomes

$$
\rho \frac{\partial Y_{i}}{\partial \tau}-\rho \frac{\chi}{2} \frac{\partial^{2} Y_{i}}{\partial Z^{2}}-\dot{m}_{i}=0
$$




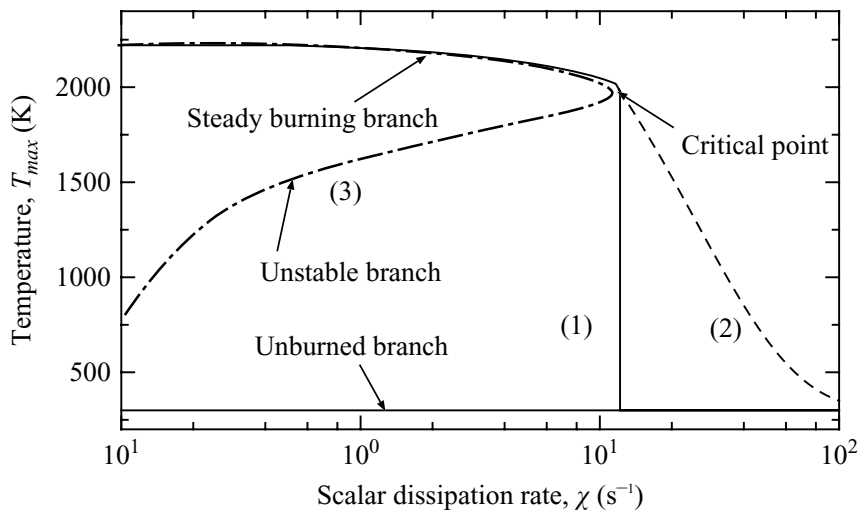

FIGURE 2. Map of maximum flamelet temperature $T_{\max }$ against scalar dissipation rate

$$
\chi=\chi_{s t} \text {. }
$$

$$
\rho \frac{\partial T}{\partial \tau}-\rho \frac{\chi}{2}\left(\frac{\partial^{2} T}{\partial Z^{2}}+\frac{1}{c_{p}} \frac{\partial c_{p}}{\partial Z} \frac{\partial T}{\partial Z}\right)+\frac{1}{c_{p}} \sum_{i=1}^{N} h_{i} \dot{m}_{i}=0 .
$$

Here, $\tau$ is the time, $c_{p}$ the specific heat at a constant pressure, $h_{i}$ the enthalpy of the $i$ th species, and $\dot{m}_{i}$ the mass production or consumption rate of the $i$ th species.

The scalar dissipation rate $\chi$ in (2.37) and (2.38) has a distribution in the $Z$ space. In the counterflow configuration, the distribution is assumed to be

$$
\chi(Z)=\frac{a_{s}}{\pi} \exp \left\{-2\left[\operatorname{erfc}^{-1}(2 Z)\right]^{2}\right\},
$$

where $a_{s}$ is the strain rate, indicating the maximum velocity gradient, and $\operatorname{erfc}^{-1}$ is the inverse of the complementary error function (Peters 1984; Pitsch \& Peters 1998). Although (2.39) has a dependence on the physical space parameter $a_{s}$, the dependence vanishes on introducing the equation for $Z=Z_{s t}$. The $\chi$ distribution as a function of $Z$ is obtained as

$$
\chi(Z)=\chi_{s t} \frac{\exp \left\{-2\left[\operatorname{erfc}^{-1}(2 Z)\right]^{2}\right\}}{\exp \left\{-2\left[\operatorname{erfc}^{-1}\left(2 Z_{s t}\right)\right]^{2}\right\}} .
$$

In SFM, the values of $Z$ and $\chi=\chi_{s t}=\chi\left(Z_{s t}\right)$ in the chemtable are supposed to be equal to those of $Z$ and $\chi$ in the flow field, respectively. Figure 2 shows the map of the maximum temperature of the flamelet $T_{\max }$ against $\chi$ obtained from the chemtable. Line (1) is the original temperature profile obtained from the steadyflamelet solution. It is found that at a critical point of $\chi, T_{\max }$ drops down from the steady burning branch to the unburned branch, since the flamelet is unable to maintain the reaction for larger $\chi$. This discontinuous jump is known to cause numerical instability and non-physical solutions in SFM. Therefore, the smoothed temperature profile should be used. Usually, the PDF to consider the SGS fluctuation of $\chi$, such as a lognormal function, acts to smooth the temperature profile in LES and RANS (Jiménez et al. 1997; Pierce \& Moin 2004). However, since DNS lacks the SGS fluctuation of $\chi$, we employ an exponential function instead of the lognormal function. The smoothed temperature prescribed by this exponential function is shown by line (2). Line (3) represents the complete solution to the flamelet equation, including an unstable branch. The profile assumes the well-known S-shape, while the lower part of the temperature profile corresponds to an unstable branch. This unstable branch is provisionally accounted for in FPVA, as shown later. 

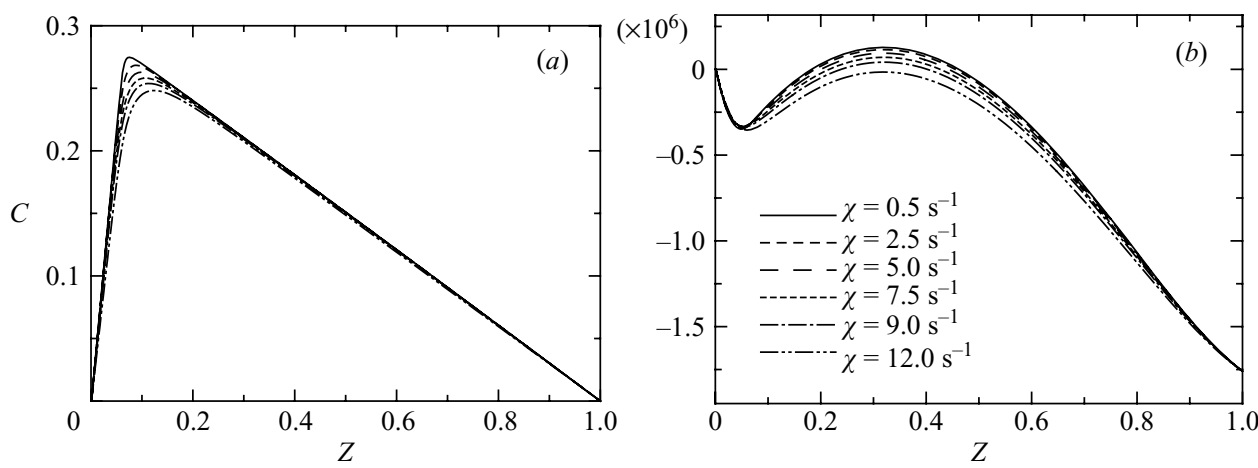

Figure 3. Profiles of progress variable $C$ in $Z$ space. (a) $C=Y_{C O 2}+Y_{H 2 O}$ and (b) $C=h$.

Concerning the reaction mechanism, a full kinetic mechanism for the fuel or its reduced version is recommended. However, to compare the results of ARF, a one-step global reaction, which is the same as (2.22), is employed here. Equations (2.37) and (2.38) are simultaneously and implicitly solved with 1000 computational grid points in $Z$ space. Differentials of variables are approximated by a second-order finite central difference method. The CHEMKIN database is also linked during the calculations to provide thermodynamics (Kee et al. 1986, 1989).

Figure 3 shows profiles of the product mass fraction, $C=Y_{\mathrm{CO} 2}+Y_{\mathrm{H} 2 \mathrm{O}}$, and the total enthalpy, $C=h$, in the $Z$ space for various scalar dissipation rates $\chi$. In both cases, $C$ is found to show uniqueness with respect to $Z$ and $\chi$, meaning that $C$ (product mass fraction or total enthalpy) and $Z$ can be used to identify other physical variables instead of $\chi$ and $Z$.

This chemtable for SFM and FPVA is incomplete for spray flames, because the heat transfer between the dispersed droplet and the carrier gaseous phase is not taken into account in this form. In spray flames, generally, the low droplet temperature tends to reduce the flame temperature, and this effect is enhanced by the heat loss due to the droplet evaporation (i.e. the droplet cooling effect, Nakamura et al. 2005). In Ham et al. (2003) and Kurose et al. (2004), the heat loss due to the droplet evaporation is accounted for by subtracting the equivalent reduced temperature directly from the gaseous temperature in the chemtable. The heat loss increases as the fuel vapour concentration rises, so that the reduced temperature can be assumed to be proportional to the fuel vapour concentration. Therefore, the temperature decrease is expressed by a linear function of $Z$ as

$$
\Delta T=-\frac{L_{V} Z}{c_{p}} .
$$

However, it should be noted that according to the governing equations, heat loss due to the droplet evaporation should reduce the droplet temperature, and the reduced droplet temperature, in turn, decreases the gaseous temperature through heat transfer at the droplet surface. Therefore, this is a tentative treatment and still does not account for the actual heat transfer between the dispersed droplets and the carrier gaseous phase.

On the other hand, when total enthalpy is chosen as the progress variable for FPVA, the problem of the value of the enthalpy in the flow field exceeding the maximum limit of that in the chemtable emerges. This is because the enthalpy in the chemtable does not account for the enthalpy increases caused by the high-temperature coflow 


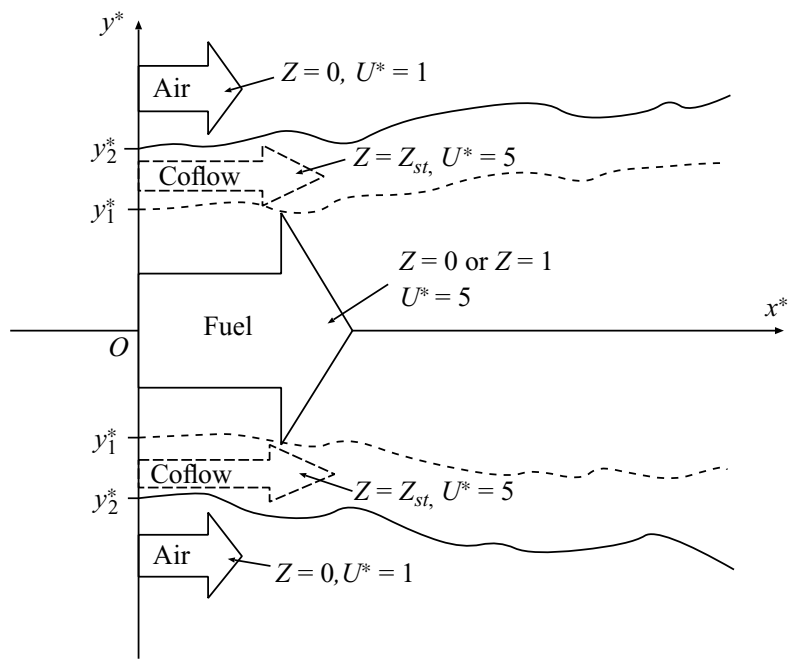

FIGURE 4. Schematic of computational domain and inlet conditions.

in flame stabilization nor the mass transfer from droplets to the gaseous phase due to the evaporation in the flow field. To take this effect into account, the temperature increase is calculated by

$$
\Delta T=\frac{C-C_{c}}{c_{p}} .
$$

This temperature modification is applied only when the enthalpy in the flow field $C$ exceeds the upper limit of that in the chemtable $C_{c}$.

The above two temperature modifications, (2.41) and (2.42), are conducted as a post-process of the chemtable generation, because these effects cannot be included in the one-dimensional flamelet equation. The gaseous temperature modified in this manner is used in the computation of the flow field. The validity of these modifications will be discussed later.

\subsection{Numerical conditions}

Numerical simulations are conducted for the gaseous and spray jet flames with the various combustion models. Figure 4 shows a schematic of the computational domain and inlet conditions. Besides the fuel supply condition, the computational domains and conditions for the gaseous and spray jet flames are almost identical.

The dimensions of the computational domain, non-dimensioned by half the spanwise width $L_{0}$, are 5 and 2 in the streamwise and spanwise directions, respectively. In this paper, length scales $x$ and $y$ non-dimensioned by $L_{0}$ are shown as $x^{*}$ and $y^{*}$, respectively, so that the non-dimensional computational domain is $0<x^{*}<5$ and $-1<y^{*}<1$. The fuel, stoichiometric mixture and air are separately issued from the inlets at $-0.05<y^{*}<0.05,-0.06<y^{*}<-0.05$ and $0.05<y^{*}<0.06$, and $y^{*}<-0.06$ and $0.06<y^{*}$, respectively. In figure 4 , this is shown by $y_{1}^{*}=0.05$ and $y_{2}^{*}=0.06$. On the other hand, $y_{1}^{*}$ and $y_{2}^{*}$ are set to be 0.065 and 0.075 respectively in the case of spray flames. The stoichiometric mixture is provided to ignite the flames, and the properties are obtained from the chemtable. The temperature of the fuel and air is set to be $T^{*}=1$, whereas the approximate temperature of the stoichiometric mixture is $T^{*}=7$, where the temperature is non-dimensioned by reference temperature $T_{0}$ as $T^{*}=T / T_{0}$. The velocities of the fuel, stoichiometric mixture and air are set to be 
$U^{*}=5, U^{*}=5$ and $U^{*}=1$, respectively, where the velocities are non-dimensioned by reference velocity $U_{0}$ as $U^{*}=U / U_{0}$. For the initial streamwise and spanwise velocities, velocity perturbations with a magnitude of $5 \%$ of the inlet velocity are imposed. The perturbations for the streamwise and spanwise velocities, $u_{1 p t}^{*}$ and $u_{2 p t}^{*}$, are based on continuous sine functions (Pitsch \& Steiner 2000; Réveillon \& Vervisch 2005) as

$$
u_{1 p t}^{*}=0.25 \sin \left(\frac{2 \pi t^{*}}{T_{1}^{*}}\right), \quad u_{2 p t}^{*}=0.25 \sin \left(\frac{2 \pi t^{*}}{T_{2}^{*}}\right),
$$

where $t^{*}$ is the time, and $1 / T_{1}^{*}=14.3$ and $1 / T_{2}^{*}=25.0$ are the frequencies of the perturbations. In this study, the reference values of $L_{0}, T_{0}$ and $U_{0}$ are $0.015 \mathrm{~m}, 300 \mathrm{~K}$ and $3 \mathrm{~m} \mathrm{~s}^{-1}$, respectively.

Gaseous evaporated fuel and spray fuel are supplied for the gaseous and spray flames, respectively. In the case of the spray flame, the carrier gas is chosen to be air $(Z=0)$. N-decane $\left(\mathrm{C}_{10} \mathrm{H}_{22}\right)$ is used as the fuel, and the liquid properties are obtained from Abramzon \& Sirignano (1989). The boiling temperature of the droplet is $T_{B L}=447.7 \mathrm{~K}$, the heat capacity is $c_{L}=2520.5 \mathrm{~J} \mathrm{~kg}^{-1} \mathrm{~K}^{-1}$ and the density is $\rho_{d}=642 \mathrm{~kg} \mathrm{~m}^{-3}$. The latent heat of the droplet $L_{V}$ is a function of the temperature and given as $L_{V}=3.958 \times 10^{4}\left(619-T_{d}\right)^{0.38} \mathrm{~J} \mathrm{~kg}^{-1}$. For the spray flame, initial droplet locations are randomly set at a streamwise distance of around $x^{*}=0$, and the velocities are set to be equivalent to the gaseous-phase velocities at the centre of the droplets. The initial non-dimensional droplet diameter $d_{d}^{*}\left(=d_{d} / L_{0}\right)$ is determined between $6.7 \times 10^{-5}$ and $6.7 \times 10^{-3}$ (mean diameter is $3.4 \times 10^{-3}$ ) by using a homogeneous droplet diameter distribution. The droplets are supplied into the domain continuously in time, and the total mass supply rate $m_{\phi}^{*}\left(=m_{\phi} \rho_{0}^{-1} L_{0}^{-2} U_{0}^{-1}\right)$ is set to be $m_{\phi}^{*}=11.1$, where $\rho_{0}$ is the reference density of $\rho_{0}=0.1 \mathrm{~kg} \mathrm{~m}^{-3}$. All physical variables are non-dimensioned by the above reference values, and the non-dimensional variables are shown with superscript asterisk hereafter.

Reynolds numbers $R e$ for the cold flows of the gaseous and spray flames differ because of the difference in properties of n-decane and air. The Reynolds numbers, estimated using of the inlet fuel jet width and the difference between the velocities of the fuel and air for the gaseous and spray flames are $R e=18000$ (n-decane base) and $R e=2340$ (air base), respectively.

The computational domain is divided into 1000 (in the $x$-direction) $\times 400$ (in the $y$-direction) non-uniform computational grid points, and fine resolution is ensured around the centre of the streamlines. The number of grid points was determined by comparing the numerical results obtained by computations with $500 \times 200,1000 \times$ 400 and $1500 \times 600$ grid points. The number of grid points in the smallest flame thickness defined by temperature gradient for the computation with $1000 \times 400$ grid points was estimated to be about 15-20, which is considered to be enough to give reliable flame behaviours in terms of the flamelet modelling. For numerical approximation of the carrier gaseous phase, discretization of nonlinear terms of the momentum equations is derived from a fourth-order fully conservative finite difference scheme (Morinishi et al. 1998; Nicoud 2000), while those of the scalars, such as enthalpy and mass fractions, are computed by a QUICK scheme (Leonard 1979). The validity of the present fourth-order finite difference scheme in combination with QUICK was verified by comparing with the results for the second-order finite difference scheme, whose accuracy has been discussed in detail by Pierce (2001) and Pierce \& Moin (2004). Other differentials are approximated by a second-order finite difference method. A fractional step method (Kim \& Moin 1985; Nicoud 2000) and 


\begin{tabular}{lllll}
\hline Case & Fuel & \multicolumn{1}{c}{ Chemtable } & Progress variable & Method \\
& & & & \\
GAR & Gas & - & - & ARF \\
GFM & Gas & unmodified & - & SFM \\
GFM-P & Gas & unmodified & $C=Y_{C O 2}+Y_{H 2 O}$ & FPVA-P \\
GFM-E1 & Gas & unmodified & $C=h$ & FPVA-E \\
GFM-E2 & Gas & modified by (2.42) & $C=h$ & FPVA-E \\
LAR & Liquid & - & - & ARF \\
LFM & Liquid & unmodified & - & SFM \\
LFMM & Liquid & modified by (2.41) & - & SFM \\
LFM-P & Liquid & unmodified & $C=Y_{C O 2}+Y_{H 2 O}$ & FPVA-P \\
LFM-E & Liquid & modified by (2.42) & $C=h$ & FPVA-E \\
\multicolumn{7}{l}{ TABLE 1. Cases and numerical conditions used in this study. } \\
\hline
\end{tabular}

a second-order explicit Runge-Kutta method are used for the time advancement of both carrier gaseous and dispersed droplet phases. The density of the flow field is evaluated by the equation of state, and the density variation is considered in the procedure of the time advancement. A convective outflow condition is applied to outflow boundary in the streamwise direction. This is given by

$$
\frac{\partial \phi}{\partial t}+u_{m} \frac{\partial \phi}{\partial x_{j}}=0 .
$$

Here, $\phi$ is a certain dependent variable, and $u_{m}$ is the convective velocity at the outflow boundary. A slip wall condition is applied in the spanwise direction.

Table 1 shows the cases and numerical conditions in this study. These cases are roughly classified into two groups, i.e. the gaseous and spray flames. The first capital letter of the case names $\mathrm{G}$ and $\mathrm{L}$ indicates the gaseous and spray flames, respectively. The following letters, AR, FM, FM-P and FM-E represent the combustion models ARF, SFM, FPVA-P and FPVA-E, respectively. The validity of the temperature modifications using (2.41) and (2.42) is also examined by comparing cases with and without the modifications respectively.

\section{Gaseous flames}

\subsection{General features}

Figure 5 shows a comparison of the instantaneous distribution of gaseous temperature $T^{*}$. In all cases, the diffusion flames are stably formed. It is observed that as the flame evolves downstream, the turbulent organized motions become marked, the flame width increases, and the low-temperature region inside the flame expands. The appearance of GFM differs greatly from that of GAR, while those of GFM-P, GFM-E1 and GFM-E2 are very similar to that of GAR. The reason why GFM fails to capture the flame behaviour is attributed to the original concept of SFM. As mentioned earlier in figure 2, since the flame temperature range of SFM is restricted to the steady burning branch (line (1)), SFM cannot account for the intermediate temperature on the unstable branch (line (3)) resulting in such a low-temperature region inside the jet flame. On the other hand, the success of GFM-P, GFM-E1 and GFM-E2 means that FPVA compensates for this weakness of SFM by introducing progress variables.

It is understand useful to the flame structure in the case of GAR before conducting comparisons of the flame statistics. The flame index FI is a useful diagnostic tool for 
(a)

(b)

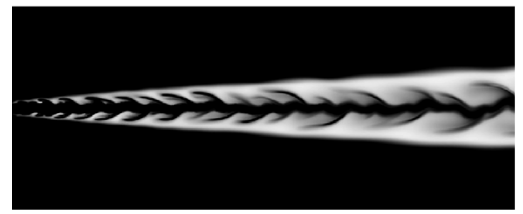

(d)

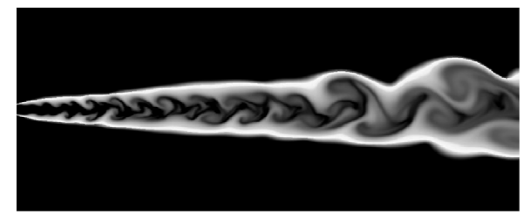

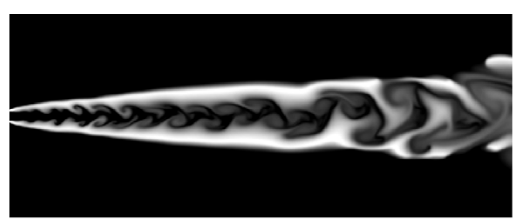

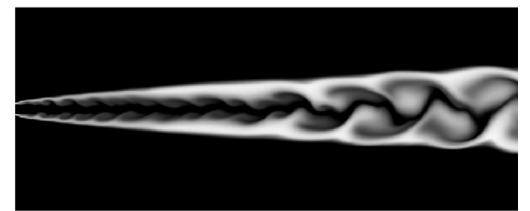

(e)

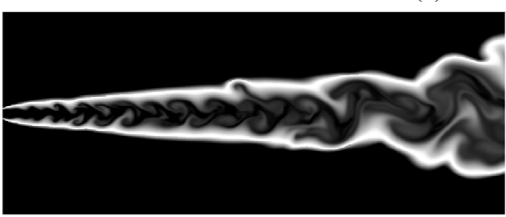

1.0 8.3

Figure 5. Comparison of instantaneous distribution of gaseous temperature $T^{*}$. (a) GAR, (b) GFM, (c) GFM-P, (d) GFM-E1 and (e) GFM-E2.

investigating the flame structure (Yamashita, Shimada \& Takeno 1996). The value of the flame index is obtained by multiplying the spatial gradients of fuel and oxidizer mass fractions as

$$
F I=\nabla Y_{V} \cdot \nabla Y_{O},
$$

where $Y_{V}$ and $Y_{O}$ are the mass fractions of fuel and oxidizer, respectively. The flame index is used to distinguish premixed and diffusion flames, respectively indicated by positive and negative values of FI. It is well known that the premixed flame appears near the inlet region of lifted diffusion jet flames for high Reynolds numbers (Domingo, Vervisch \& Bray 2002). In such flames, the mixing of fuel and oxidizer is enhanced by turbulent motions before ignition, and these flames are called partially premixed flames. Since the use of SFM and FPVA was originally limited to the diffusion flame (although Pierce \& Moin (2004) stated that FPVA is able to capture the lifted flame dynamics), the existence of the premixed flame may make it difficult to correctly validate the combustion models. However, based on figure 6 showing the instantaneous flame index distribution in the case of GAR (only the flame index distribution $T^{*}>5$ is shown), the diffusion flame is found to be dominant. This means that the effect of the premixed combustion can be neglected in the following discussion.

\subsection{Mixture fraction field}

Figure 7 shows a comparison of the instantaneous distribution of mixture fraction $Z$, at the same time as the temperature distributions in figure 5 . $Z$ issueing from the central region of the inlet is found to be diffused by the turbulent motions downstream. It is evident that the width of $Z$ in the case of GFM is much narrower than in the other cases, which, as mentioned earlier, is associated with the difference in the temperature field. 


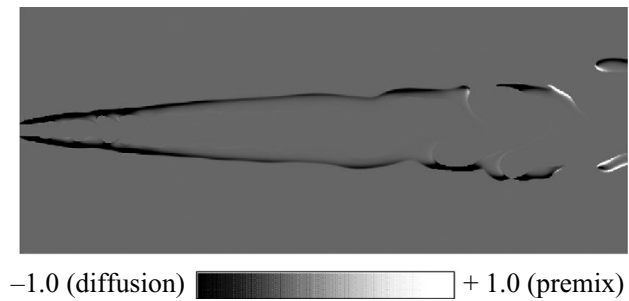

Figure 6. Distribution of flame index $F I\left(T^{*}>5\right)$ in the case of GAR.

(a)

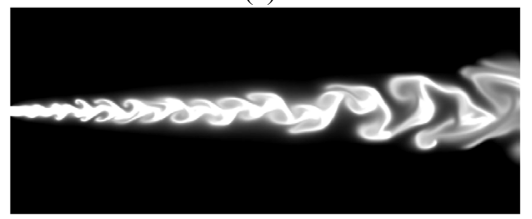

(b)

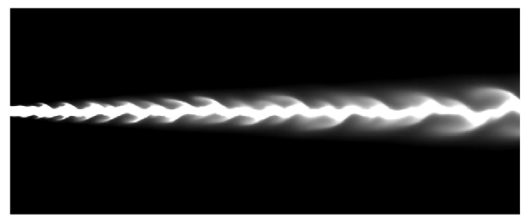

(d)

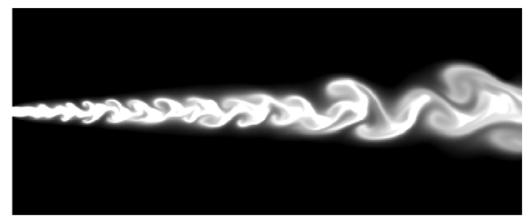

(c)

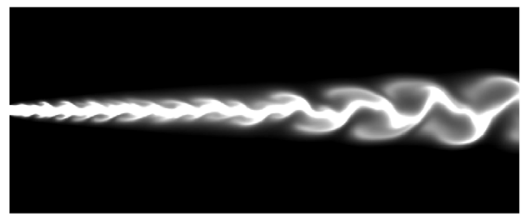

(e)

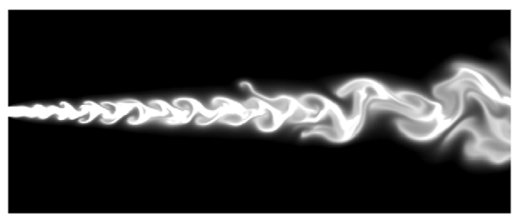

0 1.0

FIGURE 7. Comparison of instantaneous distribution of mixture fraction $Z$. (a) GAR, (b) GFM, (c) GFM-P, (d) GFM-E1 and (e) GFM-E2.

Figures 8 and 9 show comparisons of the spanwise profiles of the time-averaged mixture fraction $\bar{Z}$ and the r.m.s. of mixture fraction $Z^{\prime \prime}$, respectively. As shown in the instantaneous $Z$ distribution in figure 7, the diffusion of $Z$ in a spanwise direction is much smaller in the case of GFM than in the other cases. On the other hand, the profiles of GFM-P, GFM-E1 and GFM-E2 correlate very well with that of GAR. The r.m.s. profiles of GFM, GFM-P, GFM-E1 and GFM-E2 generally agree with that of GAR in the whole region. However, although GFM and GFM-P tend to gradually deviate from that of GAR in the downstream region beyond $x^{*}=3.0$, the profiles of GFM-E1 and GFM-E2 show a good performance.

\subsection{Scalar and gas temperature fields}

Figures 10 and 11 show comparisons of the spanwise profiles of time-averaged gaseous temperature $\overline{T^{*}}$ and r.m.s. of gaseous temperature $T^{*^{\prime \prime}}$, respectively. Clearly, GFM-E1 and GFM-E2 perform much better than GFM and GFM-P for both $\overline{T^{*}}$ and $T^{*^{\prime \prime}}$, which suggests that FPVA-E is far superior to SFM and FPVA-P. In particular, GFM and GFM-P tend to underestimate the flame width in the middle region $x^{*}=3.0$, although GFM-E1 and GFM-E2 give good predictions. It is also found that compared 

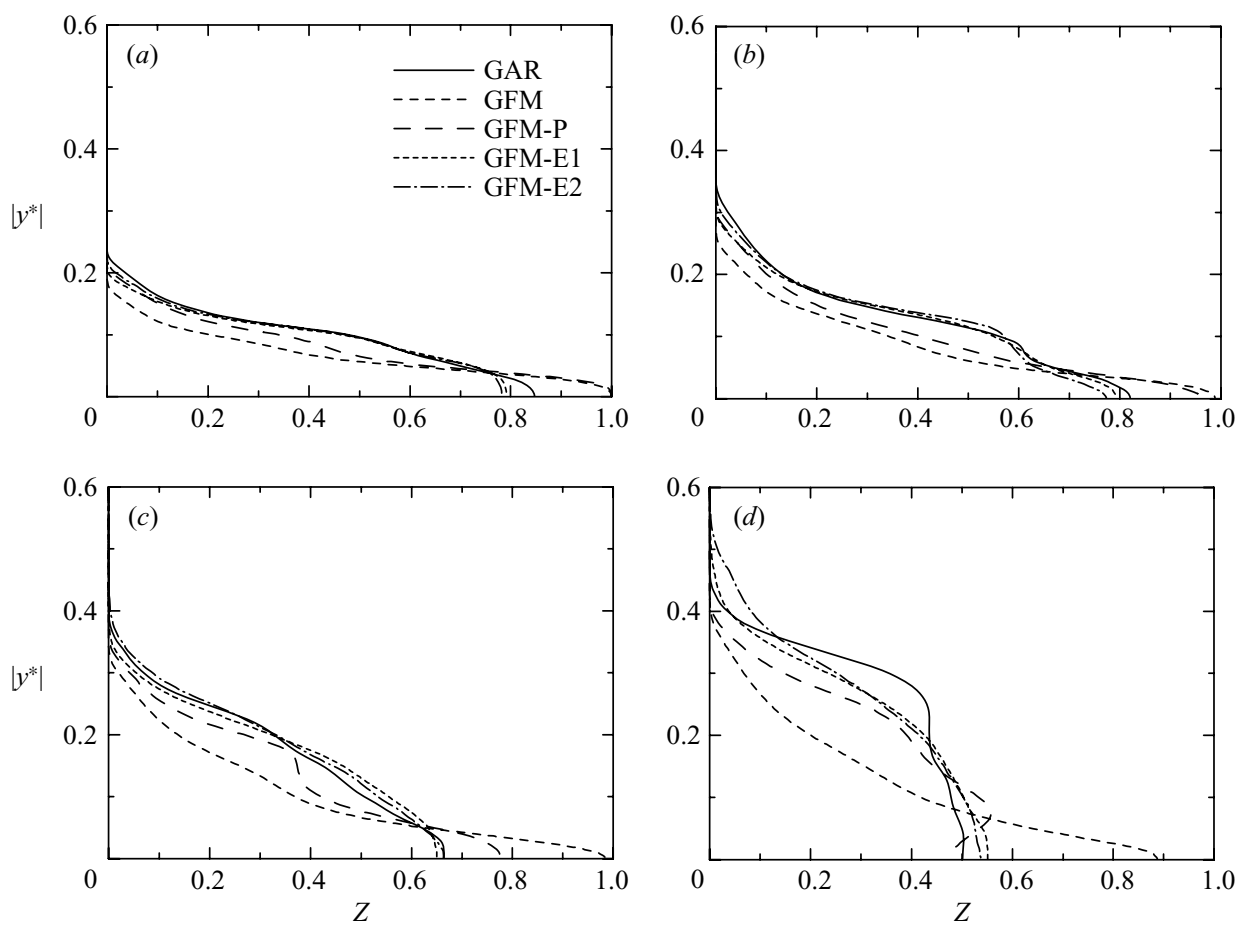

Figure 8. Comparison of spanwise profile of time-averaged mixture fraction $\bar{Z}$ at four streamwise locations. (a) $x^{*}=1.0,(b) x^{*}=2.0,(c) x^{*}=3.0$ and $(d) x^{*}=4.0$.
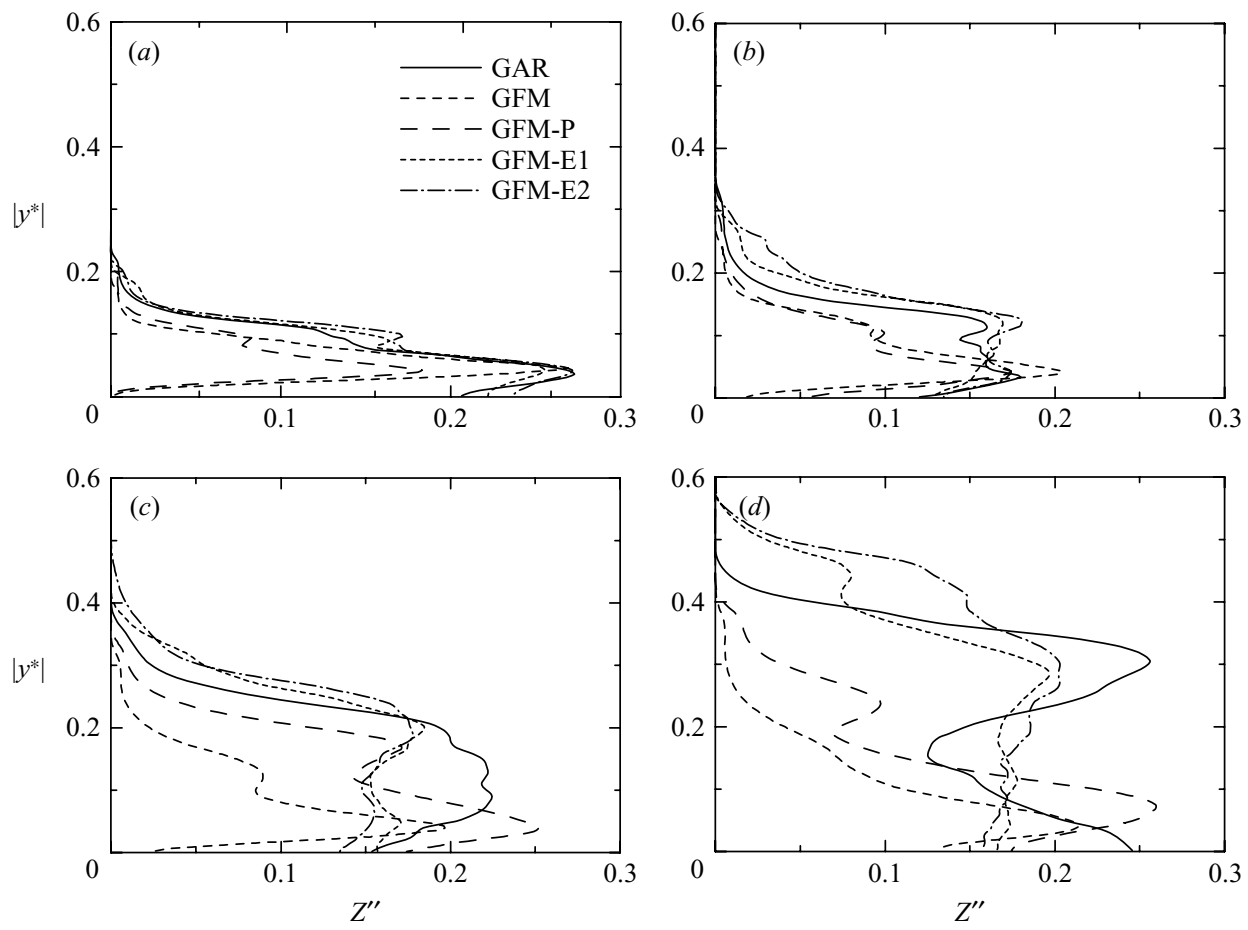

Figure 9. Comparison of spanwise profile of r.m.s. of mixture fraction $Z^{\prime \prime}$ at four streamwise locations. (a) $x^{*}=1.0,(b) x^{*}=2.0,(c) x^{*}=3.0$ and $(d) x^{*}=4.0$. 

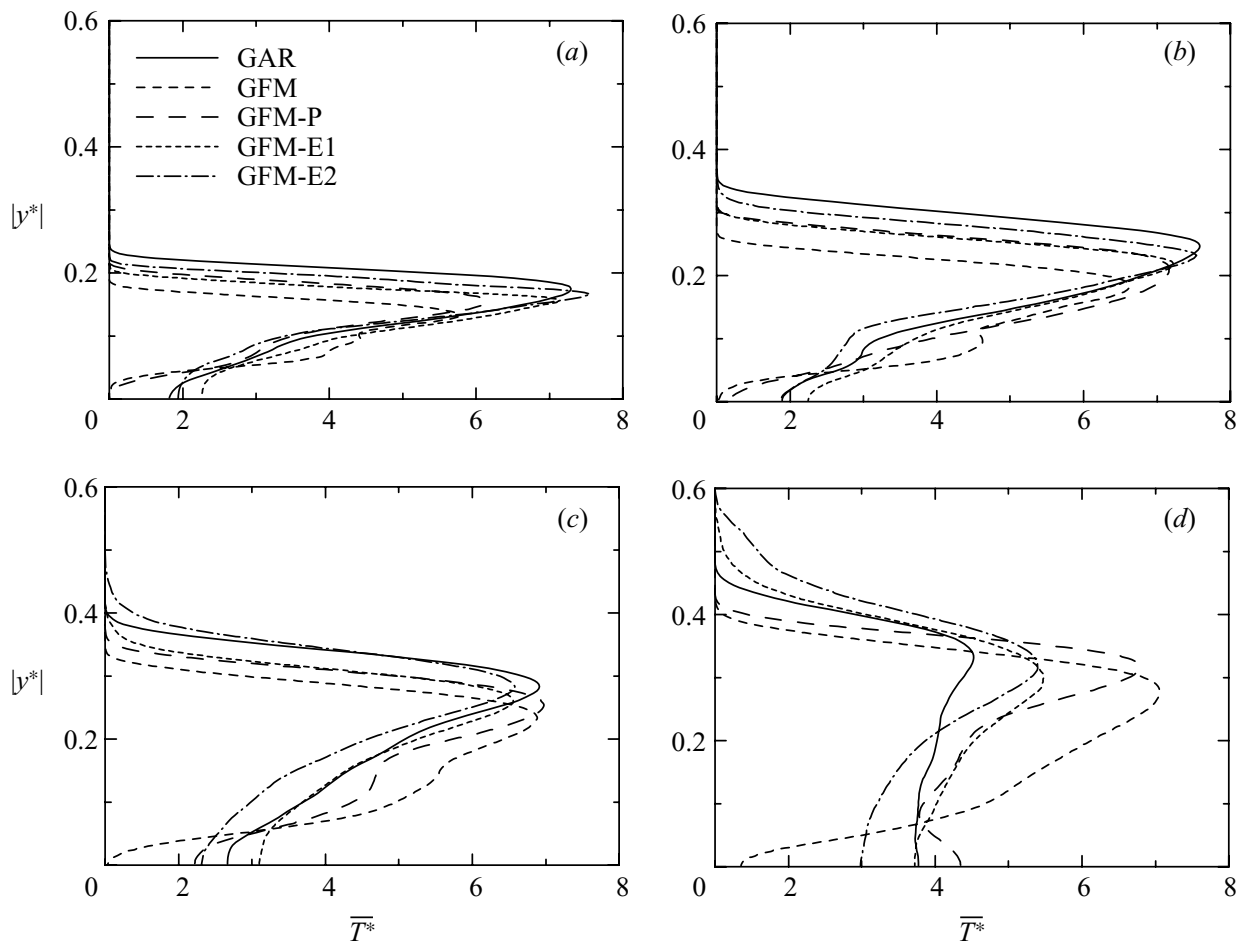

Figure 10. Comparison of spanwise profile of time-averaged gaseous temperature $\overline{T^{*}}$ at four streamwise locations. $(a) x^{*}=1.0,(b) x^{*}=2.0,(c) x^{*}=3.0$ and $(d) x^{*}=4.0$.

to GFM-E1, the profile of GFM-E2 is in better agreement with that of GAR. This is considered to be due to only FPVA-E being able to account for the hot temperature of coflow for the flame stabilization, and that the advantage becomes more marked by introducing (2.42).

To examine the predictability of the unstable branch in figure 2, scatter plots of the instantaneous gaseous temperature $T^{*}$ against the scalar dissipation rate $\chi^{*}$ are shown in figure 12. In each case, certain pairs of $T^{*}$ and $\chi^{*}$ are arbitrarily extracted from the whole region. Each pair of $T^{*}$ and $\chi^{*}$, therefore, represents various status of flamelets for all ranges of $Z$. As estimated by the scatter plots of GFM in figure 12(b), the critical point of $\chi^{*}$ for the steady burning branch and the unstable branch is approximately 0.07 . The existence of the plots for $T^{*}>1$ and $\chi^{*}>0.07$ is attributed to the exponential function employed to smooth the discontinuous temperature jump (see figure 2). It is found that in the range $0<\chi^{*}<0.07$, the plots of GAR concentrate in the lower temperature range of $2<T^{*}<4$, whereas those of GFM inconsistently concentrate in the higher temperature range of $4<T^{*}<7.5$. The upper and lower temperature ranges are considered to correspond to the steady burning and unstable branches, respectively. Therefore, this discrepancy confirms the fact that SFM cannot account for the unstable branch. As can be seen in figure $10(d)$, the $\overline{T^{*}}$ profile of GFM becomes much larger than that of GAR downstream. Since $\chi^{*}$ generally decreases downstream, this temperature increase is thought to be due to the fact that GFM refers to these original $T^{*}-\chi^{*}$ plots in the upper temperature range. On the other hand, the scatter plots of GFM-P, GFM-E1 and GFM-E2 are seen to be concentrated in the lower temperature range, which means that FPVA makes it 

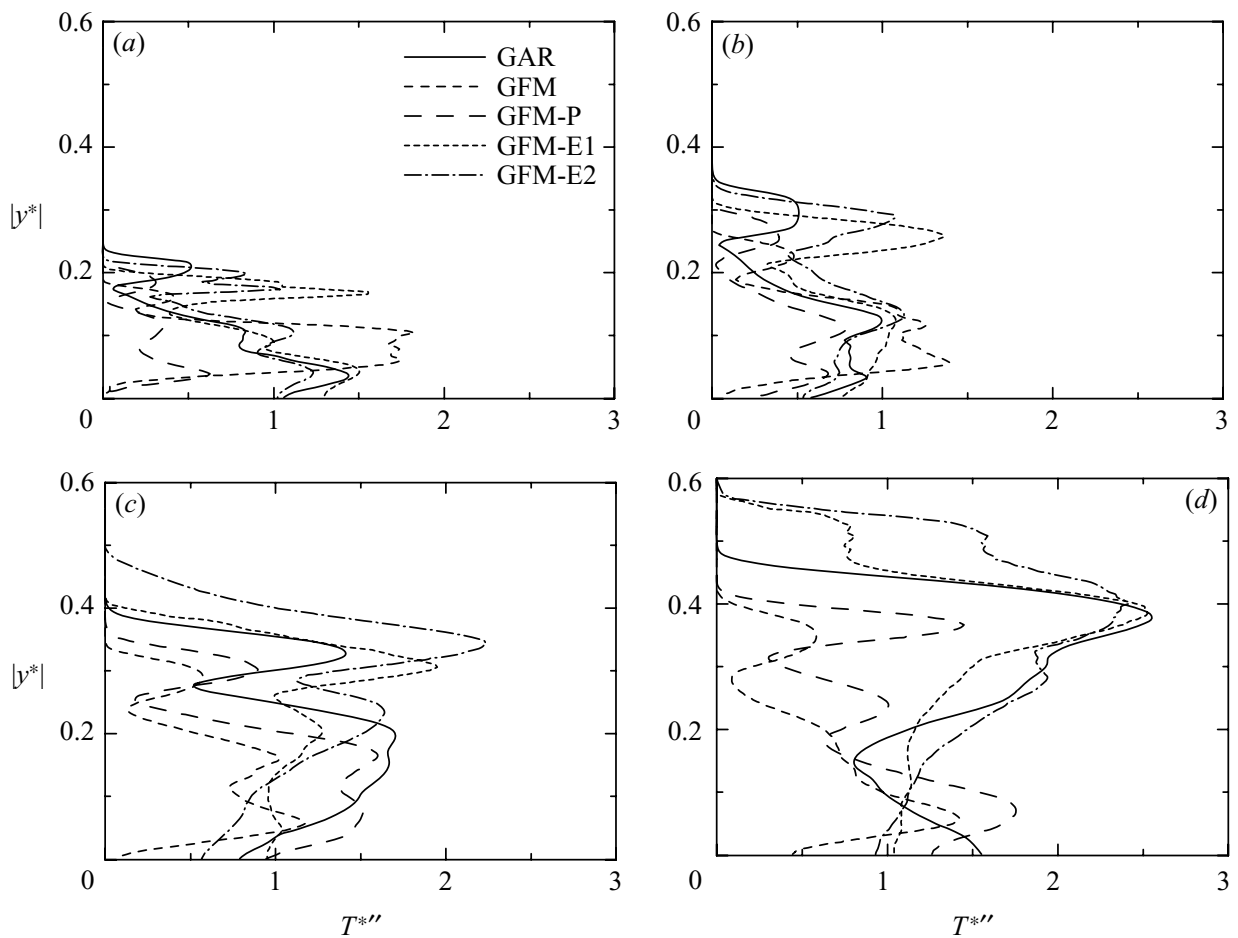

FIGURE 11. Comparison of spanwise profile of r.m.s. of gaseous temperature $T^{*^{\prime \prime}}$ at four streamwise locations. $(a) x^{*}=1.0,(b) x^{*}=2.0,(c) x^{*}=3.0$ and $(d) x^{*}=4.0$.

possible to simulate the flamelets on the unstable branch. Furthermore, in the case of GFM-E2, the lower temperature range better correlates with that of GAR than the other cases. In addition, the higher temperature plots located up to $T^{*}=8$ can be captured, which shows the validity of the enthalpy modification procedure using (2.42).

It is also found that the plots of GAR, GFM-P, GFM-E1 and GFM-E2 are distributed in the wide range of $0<\chi^{*}<0.5$ and $1<T^{*}<8$ illustrated, whereas those of GFM are non-existent high $\chi^{*}$ and high $T^{*}$, despite employing an exponential function to smooth the discontinuous temperature jump. The plots in this range originate from the high-temperature coflow issuing from the inlet to ignite the flame. The reason why GFM-P, GFM-E1 and GFM-E2 can give results in this range is that $\chi^{*}$ is represented in FPVA. In other words, FPVA has the ability to capture the effect of ignition by the high-temperature coflow.

Figure 13 shows the relation between the time-averaged gaseous temperature $\bar{T}$ * and the mixture fraction $\bar{Z}$ at two streamwise locations. In the figure, the points $\bar{Z}=0$ and $\bar{Z}=1$ correspond to the central fuel side and outer oxidizer (air) side, respectively. It is found that in all cases, the profiles at the upstream location of $x^{*}=0.5$ have peaks in the region of $0.1<\bar{Z}<0.2$, although their peak values differ. This trend is similar to that of a laminar counterflow flame in the $Z$ space (Peters 1984), which means that the flamelets exist on the steady burning branch in this upstream region (see figure 2). On the other hand, at the downstream location $x^{*}=4.5$, the profile of GAR exists in the low $Z$ region of $\bar{Z}<0.5$ and indicates a lower value than at the upstream location $x^{*}=0.5$. This flame is considered to fluctuate significantly and its flamelets exist on the unstable branch (see figure 2). 

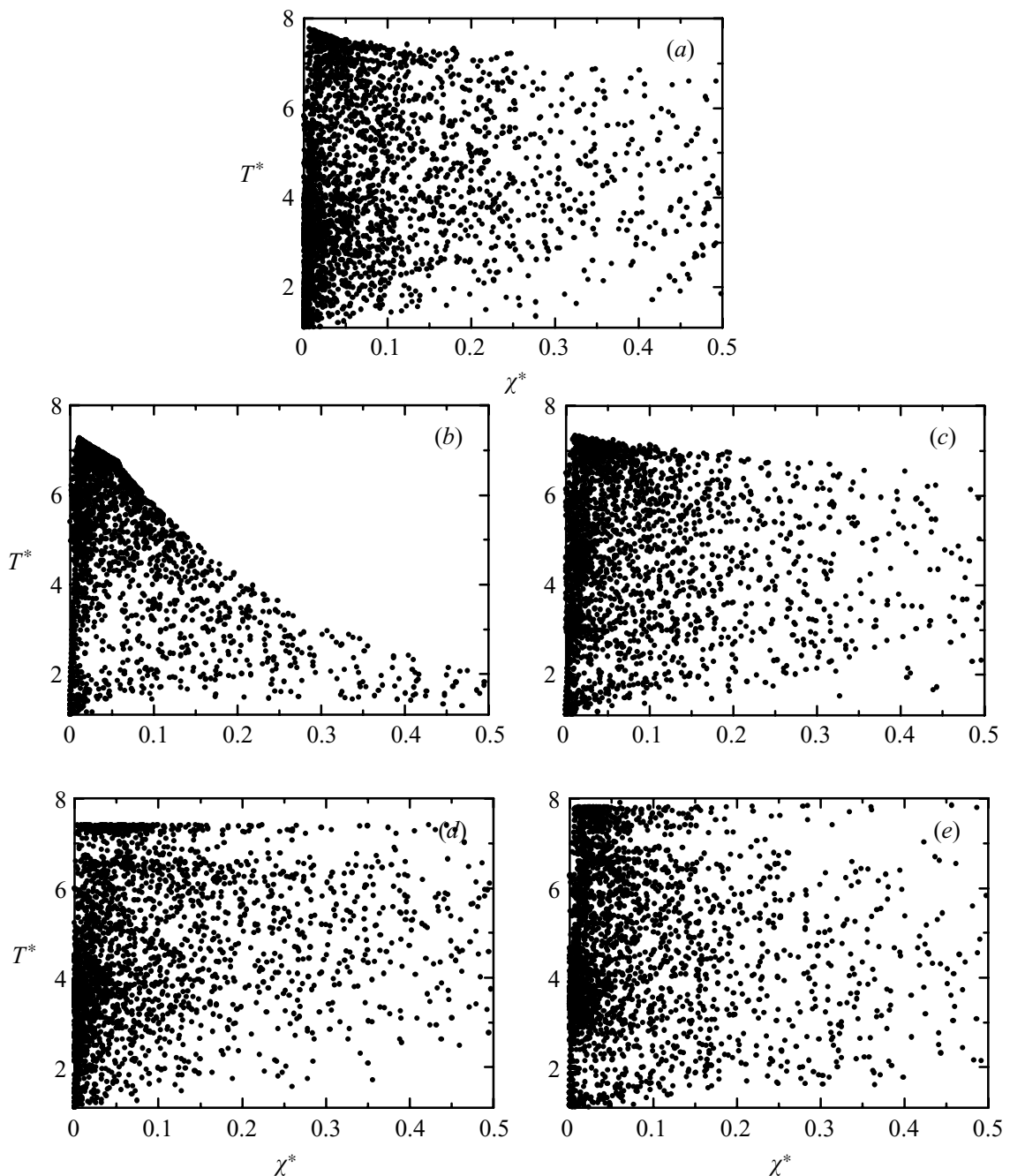

FIGURE 12. Comparison of scatter plots of instantaneous gaseous temperature $T^{*}$ against scalar dissipation rate $\chi^{*}$. (a) GAR, (b) GFM, (c) GFM-P, (d) GFM-E1 and (e) GFM-E2.

While GFM-P, GFM-E1 and GFM-E2 can predict the $\overline{T^{*}}$ decrease, GFM cannot. This is because SFM gives values only on the steady burning branch.

In summary, it can be said that FPVA provides much better results than SFM in predicting the behaviour of gaseous diffusion jet flames by capturing the unsteadiness of flamelets, and that the superiority is marked when total enthalpy is chosen as the progress variable and the enthalpy modification uses (2.42).

\section{Spray flames}

\subsection{General features}

Figure 14 shows the instantaneous features of a spray flame in the case of LAR. It is the observed that spray flame involves many simultaneous processes, such as the dispersion of fuel droplets, their evaporation, and the chemical reaction of the fuel vapour with oxidizer, and that these processes are dominated by turbulent organized 

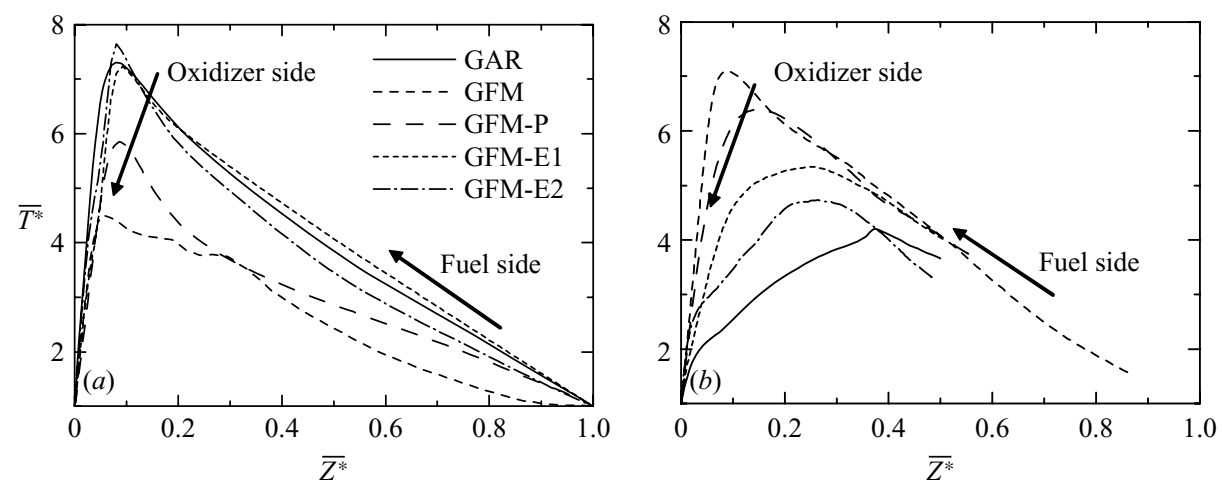

FIGURE 13. Relation between time-averaged gaseous temperature $\overline{T^{*}}$ and mixture fraction $\bar{Z}$ at two streamwise locations. (a) Upstream region $\left(x^{*}=0.5\right)$ and $(b)$ downstream region $\left(x^{*}=4.5\right)$.
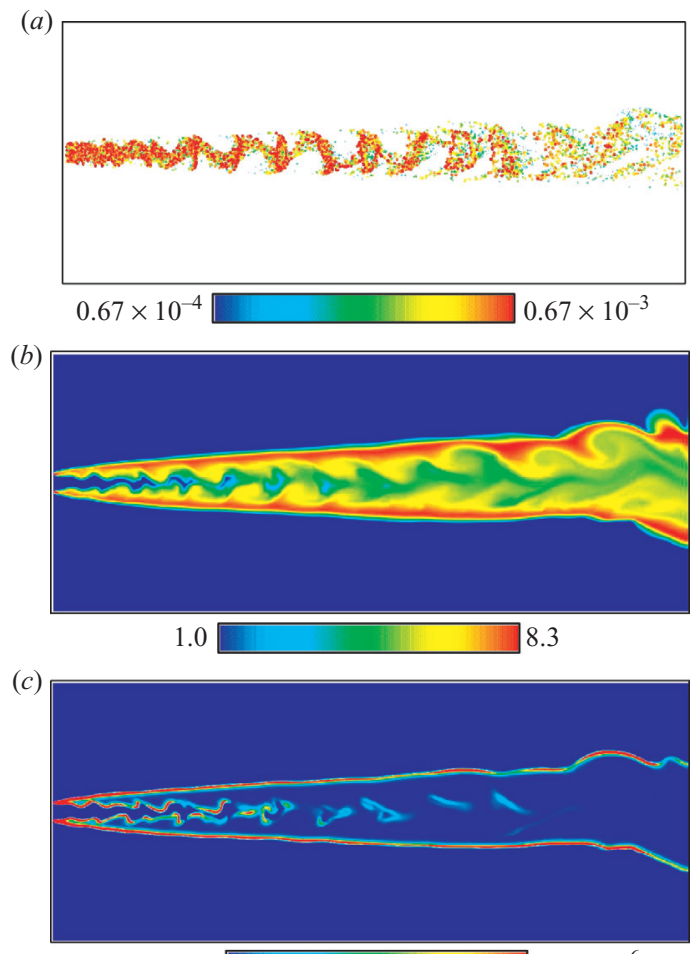

$0 \square 4.0 \times 10^{6}$

FIGURE 14. An instantaneous features of spray flame obtained in the case of LAR:

(a) droplet distribution and diameter $d_{d}^{*},(b)$ gaseous temperature $T^{*}$ and $(c)$ reaction rate $\dot{m}_{V}^{*}$.

motions. Owing to the so-called preferential motions, droplets tend to concentrate on the edges of vortices and heterogeneously evaporate, which causes differences in the flame characteristics from the gaseous flame. Unlike the gaseous flame, both the evaporated fuel and oxygen exist in the central region of the spray flame associated with droplet clusters (see also figure 18a). Consequently, the reaction takes place not 
(a)

(b)

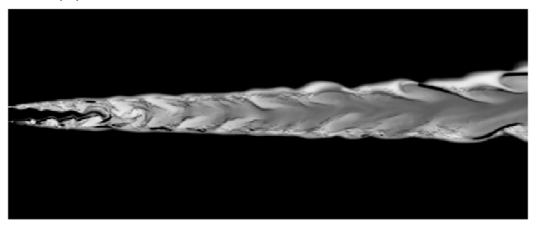

(d)

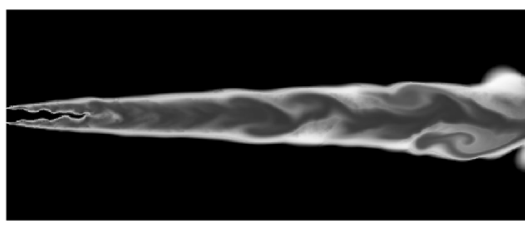

1.0
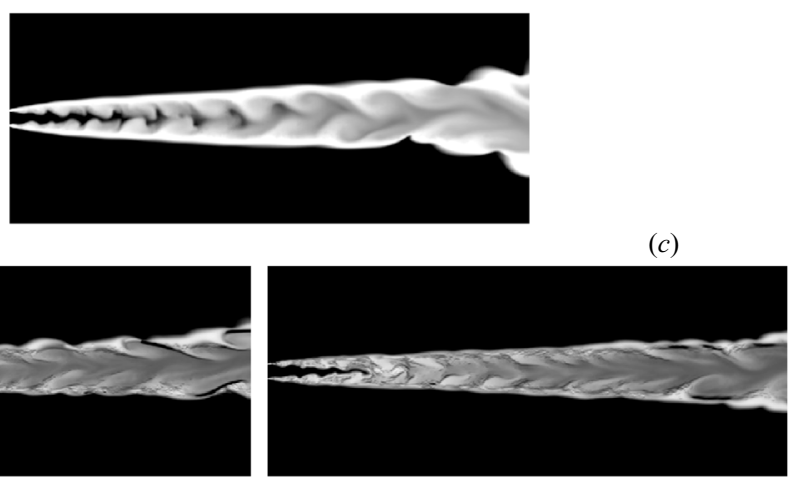

(e)

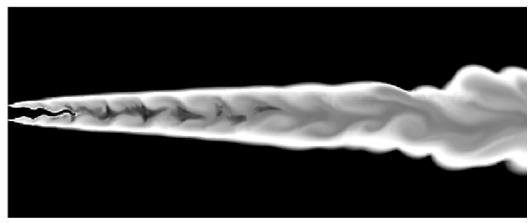

8.3

FIGURE 15. Comparison of instantaneous distribution of gaseous temperature $T^{*}$. (a) LAR, (b) LFM, (c) LFMM, (d) LFM-P and (e) LFM-E.

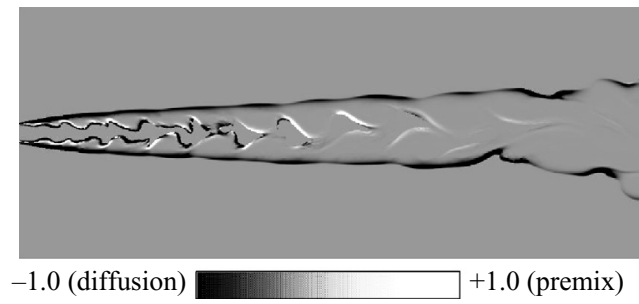

Figure 16. Distribution of flame index $F I\left(T^{*}>5\right)$ in the case of LAR.

only at the edge but also in the central region of the flame, whereas the reaction in the gaseous flame generally takes place only at the edge of the flame.

Figure 15 shows a comparison of the instantaneous distribution of the gaseous temperature $T^{*}$. In all cases, the attached spray flames are found to be stably formed owing to the high-temperature coflow. A comparison among these distributions apparently shows that LFM-E performs much better than the other cases. In particular, the high-temperature edge of the flame is well predicted in the case of LFM-E, unlike in the other cases. This is because the enthalpy increases owing to the high-temperature coflow and the mass transfer from droplets to the gaseous phase by evaporation are taken into account using (2.42).

Réveillon \& Vervisch (2005) and Domingo et al. (2005) mentioned that both premixed and diffusion flames exist in spray jet flames. As described earlier, the influence of the premixed flame should be investigated before comparing the flame statistics, since the SFM and FPVA were not originally proposed for considering the premixed flame. Figure 16 shows the distribution of the flame index $F I$ in the case of LAR. A partially premixed flame, containing both diffusion and premixed flames, 


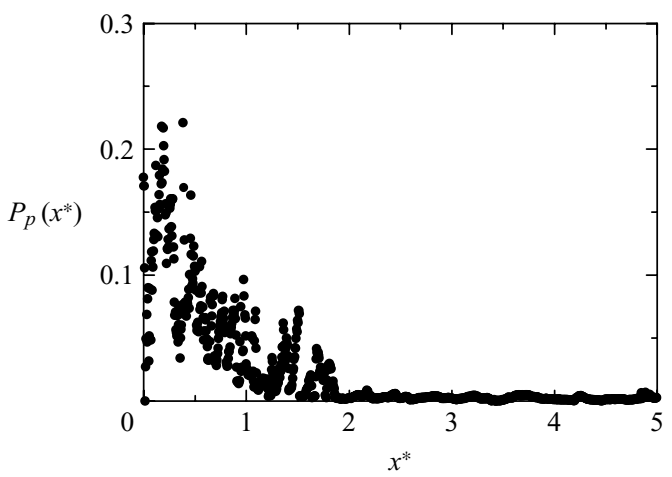

FIGURE 17. Streamwise variation of contribution of premixed flame.

is found to be formed. The premixed flames appear in the central region of the flame. Nakamura et al. (2005) demonstrated by DNS that along with the droplet trajectories, the premixed and diffusion flames appear in this order in the spray flames formed in a laminar counterflow. They also stated that these flames are caused by the premixing of the evaporated fuel and oxidizer before burning and droplet group combustion, respectively. Here, droplet group combustion is the phenomenon in which droplets burn as a group surrounded by a flame under conditions of a lack of oxygen (Chiu \& Liu 1977; Chiu, Kim \& Croke 1982). A similar trend is observed in the present spray jet flame, although it is less evident than that in the counterflow because of the turbulent organized motions and droplet preferential motions. In other words, the characteristics of the spray jet flame change from premixed-diffusion coexistent to diffusion-flame dominant downstream. This flame transition is confirmed in figure 17, which shows the streamwise variation of the contribution of the premixed flame $P_{p}$, given as

$$
P_{p}\left(x^{*}\right)=\frac{\int \dot{\omega}_{p}\left(x^{*}, y^{*}\right) \mathrm{d} y^{*}}{\int \dot{\omega}\left(x^{*}, y^{*}\right) \mathrm{d} y^{*}},
$$

where $\dot{\omega}_{p}$ and $\dot{\omega}$ are the reaction rates of the premixed flame and the sum of the premixed and diffusion flames (total reaction rate), respectively. The contribution of the premixed flame is found to exceed $20 \%$ in the upstream region, but clearly decreases and vanishes downstream.

In figure 18, the droplet positions and mass fractions of fuel and oxidizer at the same moment are shown in the case of LAR $\left(0<x^{*}<5\right)$. Only droplets existing in the high-temperature region of $T^{*}>5$ are plotted, for different evaporation rate, i.e. $\left|\dot{m}_{d}^{*}\right|>1.5 \times 10^{-3}$ or $\left|\dot{m}_{d}^{*}\right|<1.5 \times 10^{-4}$. It is found that in the downstream diffusion-flame-dominant region of $4<x^{*}<5$, the mass fractions of fuel and oxygen indicate high and low values, respectively, and many droplets with a low evaporation rate exist in the central region (see figure 18b). This behaviour corresponds to droplet group combustion. On the other hand, the diffusion flames at the edge of the flame in the upstream region, which often contain droplets with a high evaporation rate, are considered to consist of the single droplet combustion (see figure 18a).

Based on these results, the spray jet flame is a partially premixed flame, so the existence of the premixed flame may adversely affect the accuracy of the flame statistics obtained by using SFM and FPVA. 
(a)

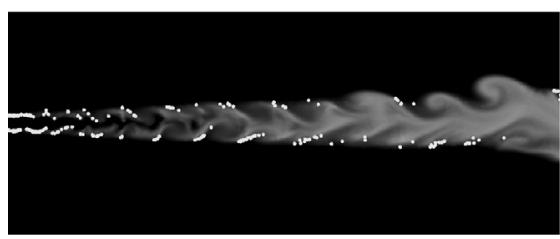

0 1.0 $Y_{v}$

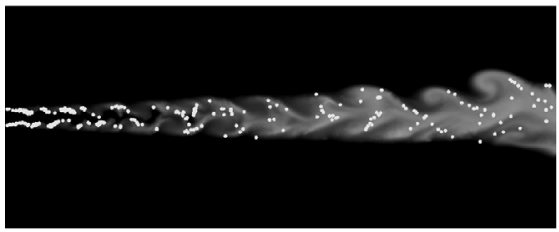

0

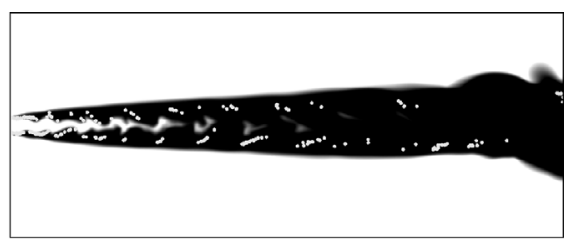

0 0.238

(b)

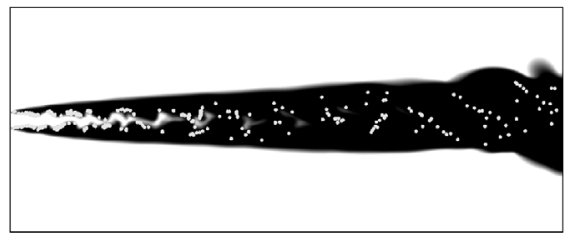

0.238

FIGURE 18. Relation between droplet positions and mass fractions of fuel and oxidizer in the case of LAR. (a) $\left|\dot{m}_{d}^{*}\right|>1.5 \times 10^{-3}$ and $(b)\left|\dot{m}_{d}^{*}\right|<1.5 \times 10^{-4}$.

(a)

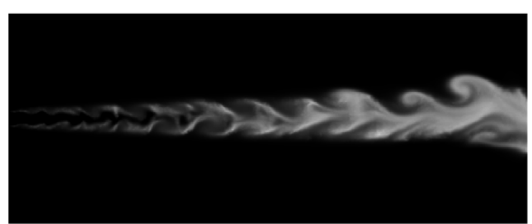

(b)

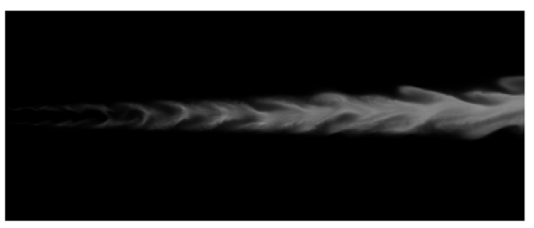

$(d)$

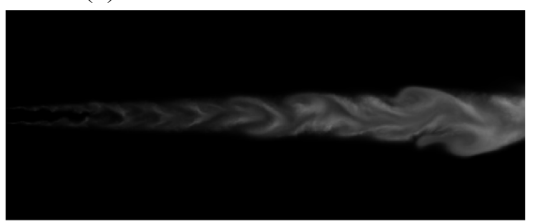

0 (c)

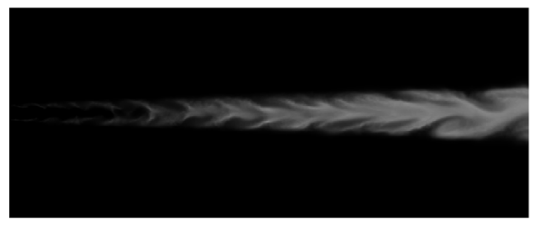

(e)

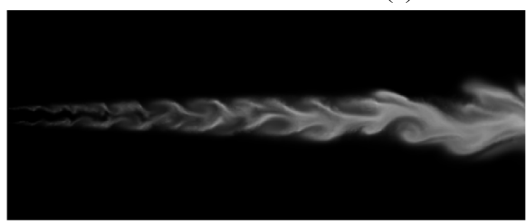

1.0

FIGURE 19. Comparison of instantaneous distribution of mixture fraction $Z$. (a) LAR, (b) LFM, (c) LFMM, (d) LFM-P and (e) LFM-E.

\subsection{Mixture fraction field}

Figure 19 shows a comparison of the instantaneous distribution of mixture fraction $Z$. In all cases, $Z$ emerges at the inlets of the high-temperature coflow and increases downstream. This trend is quite different from that in the gaseous flame, in which $Z$ emerged from the central fuel region with the initial value of unity and decreased 

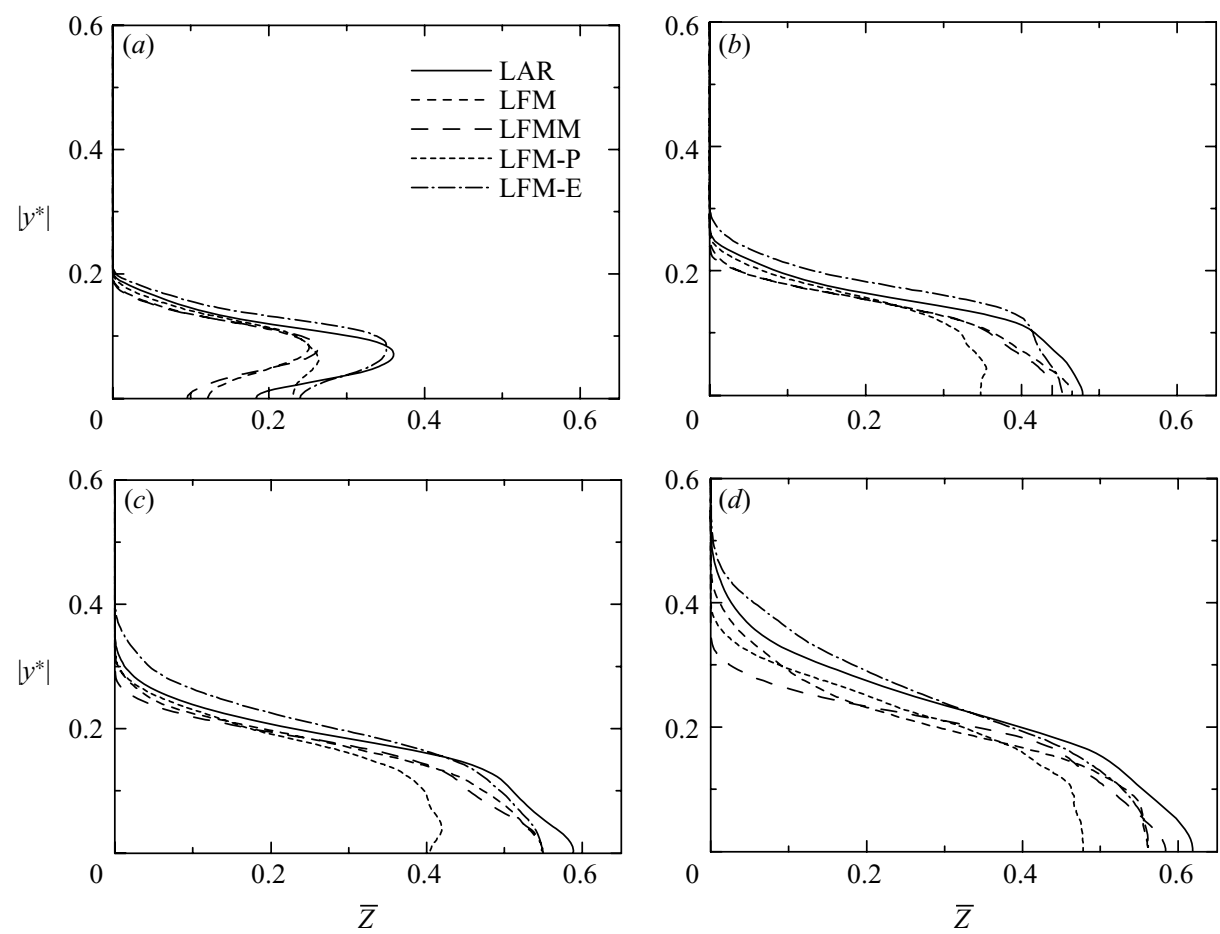

FIGURE 20. Comparison of spanwise profile of time-averaged mixture fraction $\bar{Z}$ at four streamwise locations. $(a) x^{*}=1.0,(b) x^{*}=2.0,(c) x^{*}=3.0$ and $(d) x^{*}=4.0$.

downstream, as shown in figure 7. This difference is attributed to the difference in the definition of $Z$. While $Z$ is a conserved scalar in the gaseous flame, it is a nonconserved scalar transferred from the dispersed phase by droplet evaporation in the spray flame. It is also found that LFM, LFMM, LFM-P and LFM-E underestimate the value of $Z$ in the case of LAR in the mid to downstream region. This is considered attributable to the fact that premixed flames, which cannot originally be targeted by SFM and FPVA, exist in the actual spray flame. As can be seen in figures 14, 16 and $19, Z$ increases in the central region of the flame, where the gaseous temperature is increased and the droplet evaporation is enhanced by the premixed flames.

Figures 20 and 21 show comparisons of the spanwise profiles of the time-averaged mixture fraction $\bar{Z}$ and the r.m.s. of mixture fraction $Z^{\prime \prime}$, respectively. As mentioned earlier, due to restrictions of the SFM and FPVA models, the values of $\bar{Z}$ and $Z^{\prime \prime}$ are underestimated by LFM, LFMM, LFM-P and LFM-E, especially in the mid to downstream region. Nevertheless, the profiles of LFM-E are observed to be in much better agreement with those of LAR than those of the other cases. The superiority of LFM-E is marked in the upstream region. The details will be discussed below.

It is also found that, unlike the gaseous flame, $\bar{Z}$ in the spray flame has the same value in the fuel and oxidizer streams at a fixed streamwise location in the upstream region. For example, as can be seen in figure $20(a), \bar{Z}$ in the case of LAR is 0.3 at the two spanwise locations of $\left|y^{*}\right|=0.05$ and 0.1 in the fuel and oxidizer streams, respectively. Watanabe et al. $(2007,2008)$ suggested that this behaviour may make it difficult to apply the flamelet concept to spray flames, because variables in the flow field such as gaseous temperature and chemical species mass fractions cannot be identified by $Z$ and its gradient $\chi$ alone. The details will be described below, too. 

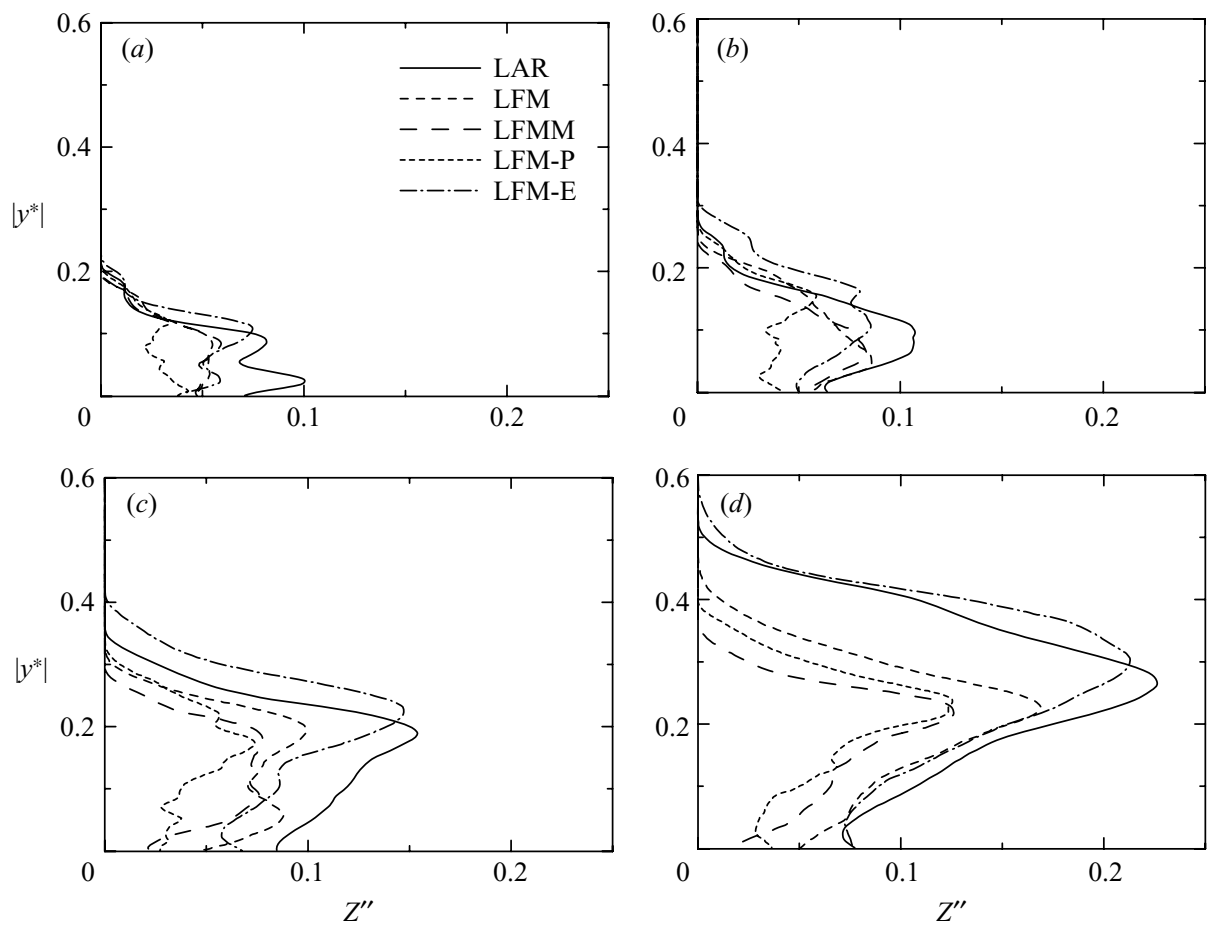

FIgURE 21. Comparison of spanwise profile of r.m.s. of mixture fraction $Z^{\prime \prime}$ at four streamwise locations. $(a) x^{*}=1.0,(b) x^{*}=2.0,(c) x^{*}=3.0$ and $(d) x^{*}=4.0$.

\subsection{Scalar and gas temperature fields}

Figures 22 and 23 show comparisons of spanwise profiles of the time-averaged gaseous temperature $\overline{T^{*}}$ and r.m.s. of the gaseous temperature $T^{* \prime \prime}$ respectively. Because of underestimation of the value of $\bar{Z}$, as mentioned earlier, the value of $\overline{T^{*}}$ is also underestimated by LFM, LFMM, LFM-P and LFM-E, especially in the centre of the mid to downstream region, compared to LAR. Comparisons among these cases show that, as also seen in the gaseous flame, the application of LFM-E is superior to the others. Even LFMM, in which the variables from the chemtable are modified by (2.41) to consider the heat loss due to the droplet evaporation, and LFM-P do not improve the accuracy very much. Although compared to the case of LFM, the maximum gaseous temperature of LFMM tends to slightly approach that of LAR downstream, significant deviation of the flame width occurs. The reason why the maximum gaseous temperature increases despite the heat loss is considered due to the fact that the heat loss slightly decreases the gaseous temperature and subsequently the evaporation rate in the central region of $0<\left|y^{*}\right|<0.1$, which induces their increases in the outer region of $0.2<\left|y^{*}\right|<0.3$. On the other hand, LFM-E improves the accuracy of the whole region due to the use of total enthalpy as a progress variable and the enthalpy modification by (2.42). Similar trends are observed in the r.m.s. profiles. The r.m.s. profile of LFM-E generally agrees with that of LAR in the whole region.

Comparisons of the time-averaged profiles of mass fractions of oxygen $\overline{Y_{O 2}}$ and carbon dioxide $\overline{Y_{\mathrm{CO} 2}}$ are shown in figure 24. It is found that not only LFM-E but also LFM-P predict the mass fraction characteristics of LAR much better than the other 

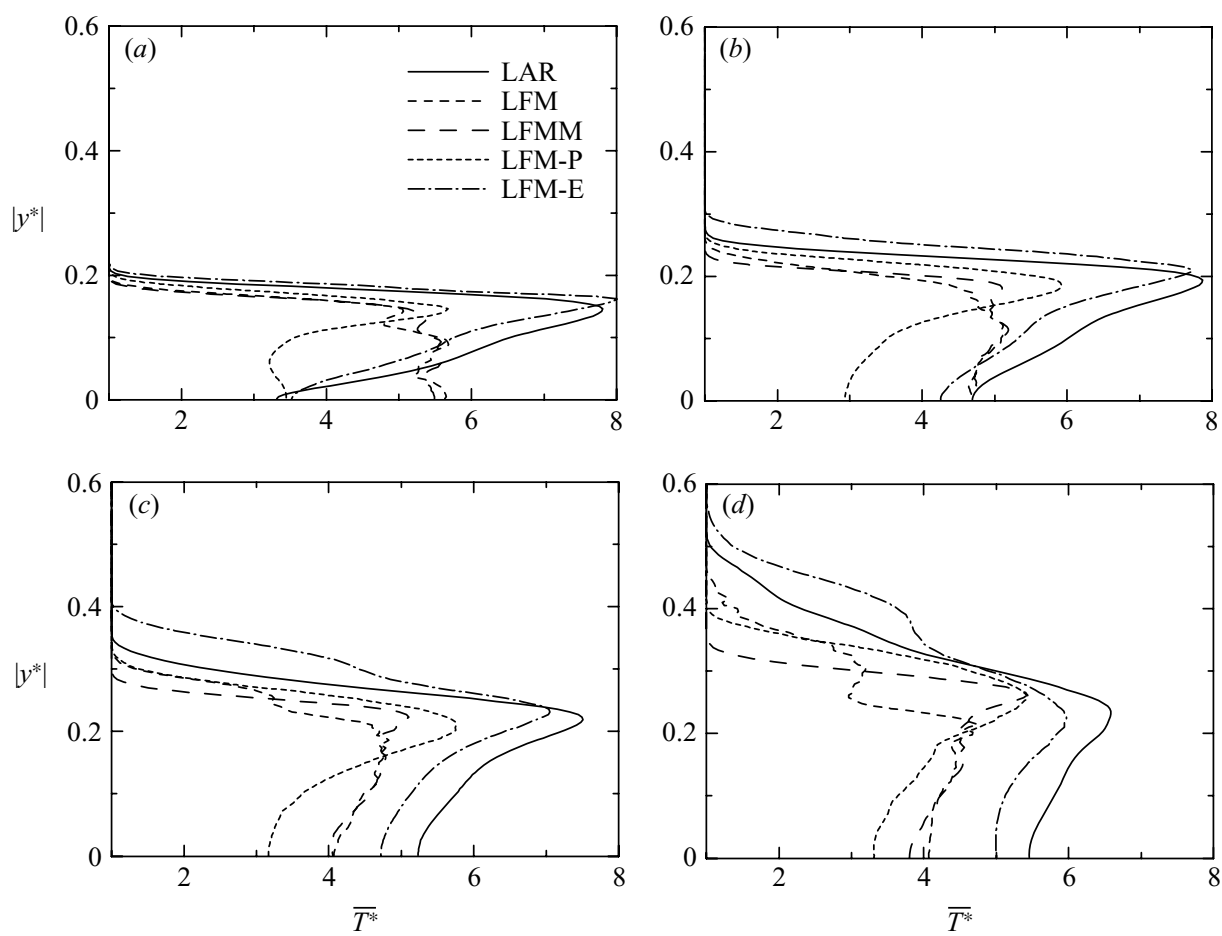

FIGURE 22. Comparison of spanwise profile of time-averaged gaseous temperature $\overline{T^{*}}$ at four streamwise locations. $(a) x^{*}=1.0,(b) x^{*}=2.0,(c) x^{*}=3.0$ and $(d) x^{*}=4.0$.

cases. This suggests that the high-temperature coflow and heat transfer with dispersed droplets do not affect the characteristics of the mass fraction field very much.

Instantaneous scatter plots of temperature $T^{*}$ against the scalar dissipation rate $\chi^{*}$ for the spray flames are shown in figure 25. In each case, some pairs of $T^{*}$ and $\chi^{*}$ are arbitrarily extracted from the whole region to represent various states of flamelets for all ranges of $Z$. It emerges that the unstable branch, which appears in the range of $2<T^{*}<4$ for the gaseous flame of GAR (see figure 12), is unclear for the spray flame of LAR. In the low $\chi^{*}$ range of $\chi^{*}<0.07$, the plots of LAR are concentrated in the range of $4<T^{*}<6$, and this trend is also seen in the other cases. The reason why the unstable branch is unclear in the spray flame is considered to be as follows. In general gaseous flames, the value of the mixture fraction $Z$ decreases downstream due to its diffusion and flamelets on the unstable branch are generated in this low- $Z$ and low $-\chi^{*}$ condition. In the present computational domain of the spray flame, however, this low- $Z$ and low- $\chi^{*}$ condition is not achieved, since $Z$ increases downstream due to the droplet evaporation. Similar flamelets on the unstable branch are considered to appear in the region further downstream of the spray flame, where the droplet evaporation finishes and the $Z$ behaviour is dominated by its diffusion. It is also found that LFM-P and LFM-E somewhat improve the prediction of the scatter plots in the high $-\chi^{*}$ and high $-T^{*}$ range by representing $\chi^{*}$ in FPVA. Furthermore, the performance is much better in the case of LFM-E, because the enthalpy increases due to the high-temperature coflow and the mass transfer from the droplets to the gaseous phase by the evaporation is considered.

Figure 26 shows the relations between the time-averaged gaseous temperature $\overline{T^{*}}$ and mixture fraction $\bar{Z}$ and between the time-averaged scalar dissipation rate $\overline{\chi^{*}}$ and 

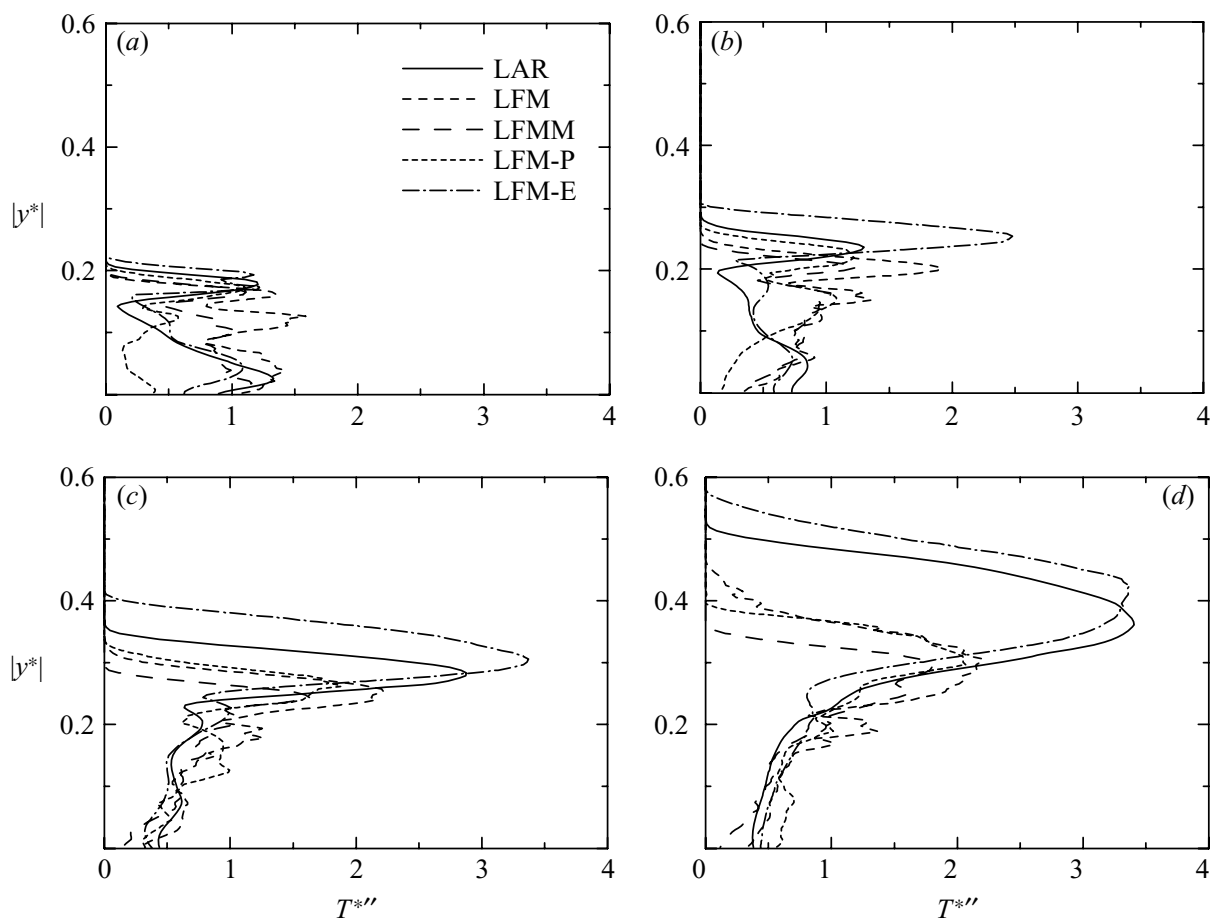

FIGURE 23. Comparison of spanwise profile of r.m.s. of gaseous temperature $T^{* \prime \prime}$ at four streamwise locations. $(a) x^{*}=1.0,(b) x^{*}=2.0,(c) x^{*}=3.0$ and $(d) x^{*}=4.0$.

the mixture fraction $\bar{Z}$ at two streamwise locations. Here, $\chi^{*}$ is directly estimated using (2.24). For the gaseous flame, the points $\bar{Z}=0$ and $\bar{Z}=1$ correspond to the central fuel side and outer oxidizer (air) side, respectively, as mentioned earlier for figure 13. At the upstream location of $x^{*}=0.5$ in the spray flame, however, this relation no longer holds (see figure $26 a$ ). It is found that $\overline{T^{*}}$ and $\overline{\chi^{*}}$ of LAR show irregular behaviours with respect to $\bar{Z}$ and tend to have two different values at a certain $\bar{Z}$. This is because in the spray flame, the mixture fraction $Z$ emerges at the inlets of the high-temperature coflow between the fuel and oxidizer streams and increases downstream (see figures 19 and 20) therefore the value of $Z$ increases and then decreases with increasing $\left|y^{*}\right|$ at a fixed streamwise location in the upstream region. This behaviour is also observed for the profiles of LFM, LFMM, LFM-P and LFM-E.

It is also found that at the upstream location of $x^{*}=0.5$, the value of $\overline{T^{*}}$ of LAR is clearly lower on the fuel side than that on the oxidizer side at a fixed $\bar{Z}$. This is considered due to the fact that the flame temperature on the fuel side is greatly reduced by the heat transfer between the dispersed droplet and the carrier gaseous phases. In spray flames, generally, the low droplet temperature tends to reduce the flame temperature, and this effect is enhanced by the heat loss due to the droplet evaporation (i.e. the droplet cooling effect, Nakamura et al. 2005; Watanabe et al. 2007, 2008). This behaviour is not predicted well by LFM and LFMM, in which SFM is employed. In SFM the value of $\bar{T}^{*}$ is determined only from the values of $\bar{Z}$ and $\overline{\chi^{*}}$ using the chemtable. Hence, it is inevitable that $\overline{T^{*}}$ has virtually the same value on the fuel and oxidizer sides in the range where the values of $\bar{Z}$ and $\overline{\chi^{*}}$ on both sides are close (i.e. $0.15<\bar{Z}<0.20$ ). Moreover, the deficiency is not recovered in FPVA-P 
(a)
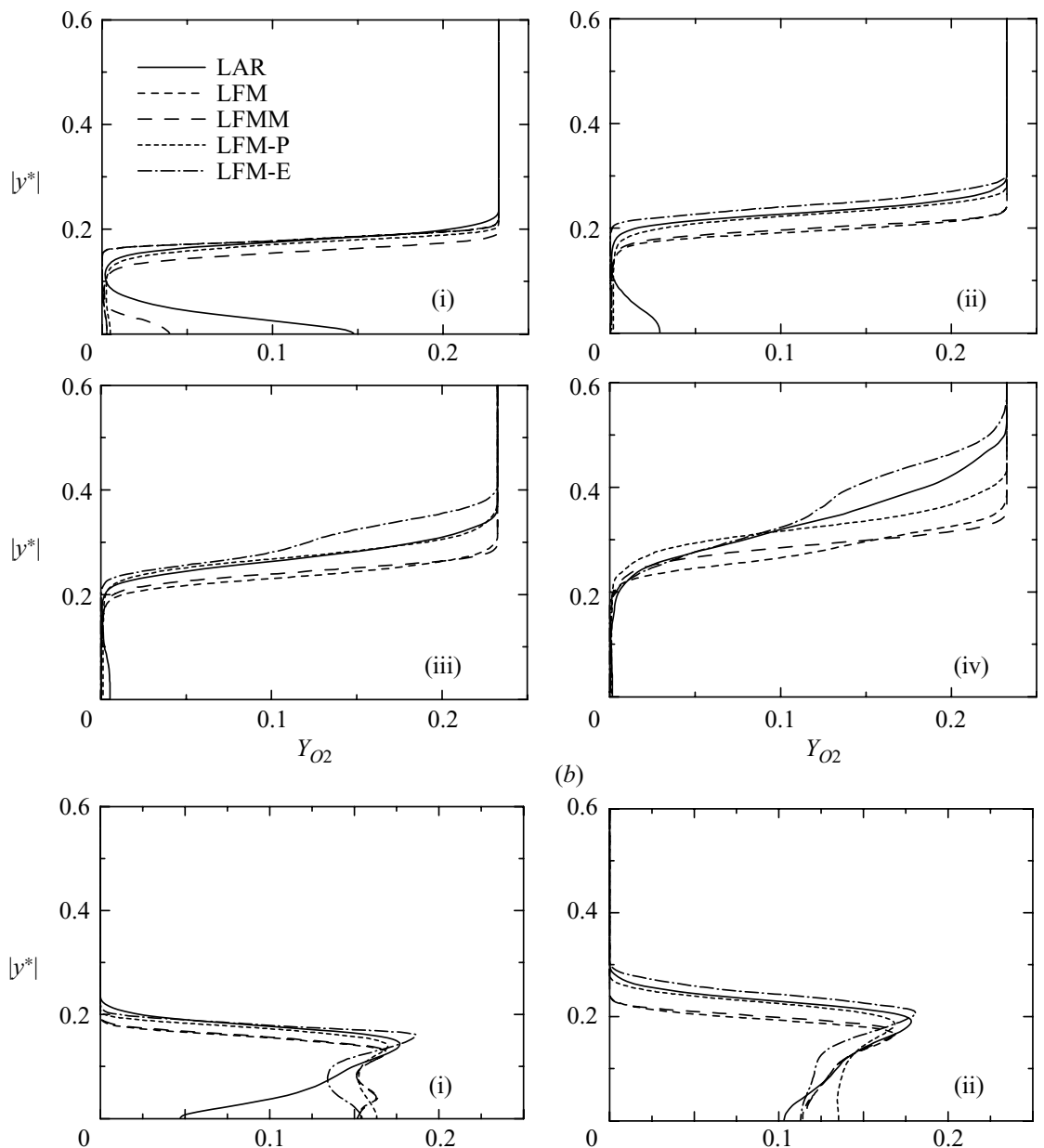

(b)
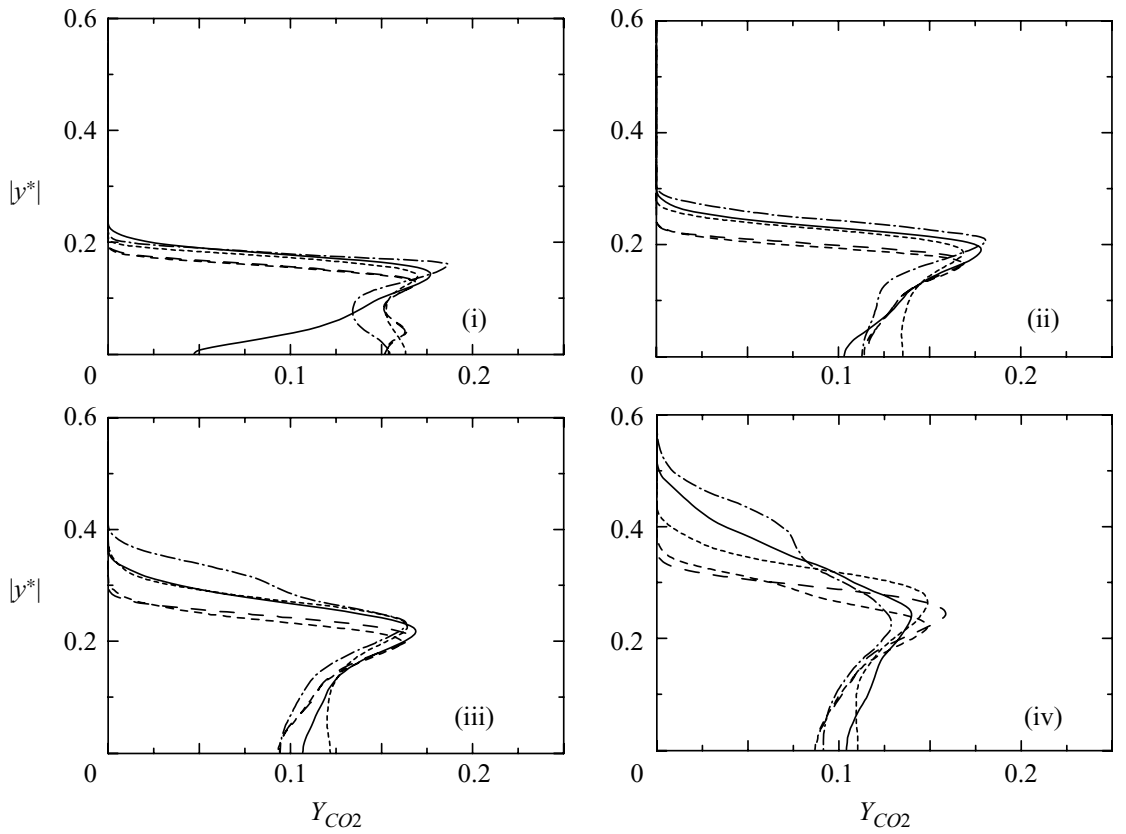

FIGURE 24. Comparisons of spanwise profiles of time-averaged $(a)$ oxygen mass fraction $\overline{Y_{O 2}}$ and $(b)$ carbon dioxide mass fraction $\overline{Y_{\mathrm{CO} 2}}$ at four streamwise locations. (i) $x^{*}=1.0$, (ii) $x^{*}=2.0$, (iii) $x^{*}=3.0$, and (iv) $x^{*}=4.0$.

as expected. Similarly to LFM and LFMM, $\overline{T^{*}}$ of LFM-P has the same value on the fuel and oxidizer sides in the range where the values of $\bar{Z}$ and $\overline{\chi^{*}}$ on both sides are identical (i.e. $0.15<\bar{Z}<0.20$ ), and does not reveal as great a temperature difference as that in the case of LAR. This means that the representation of $\overline{T^{*}}$ by $\overline{\chi^{*}}$ using 

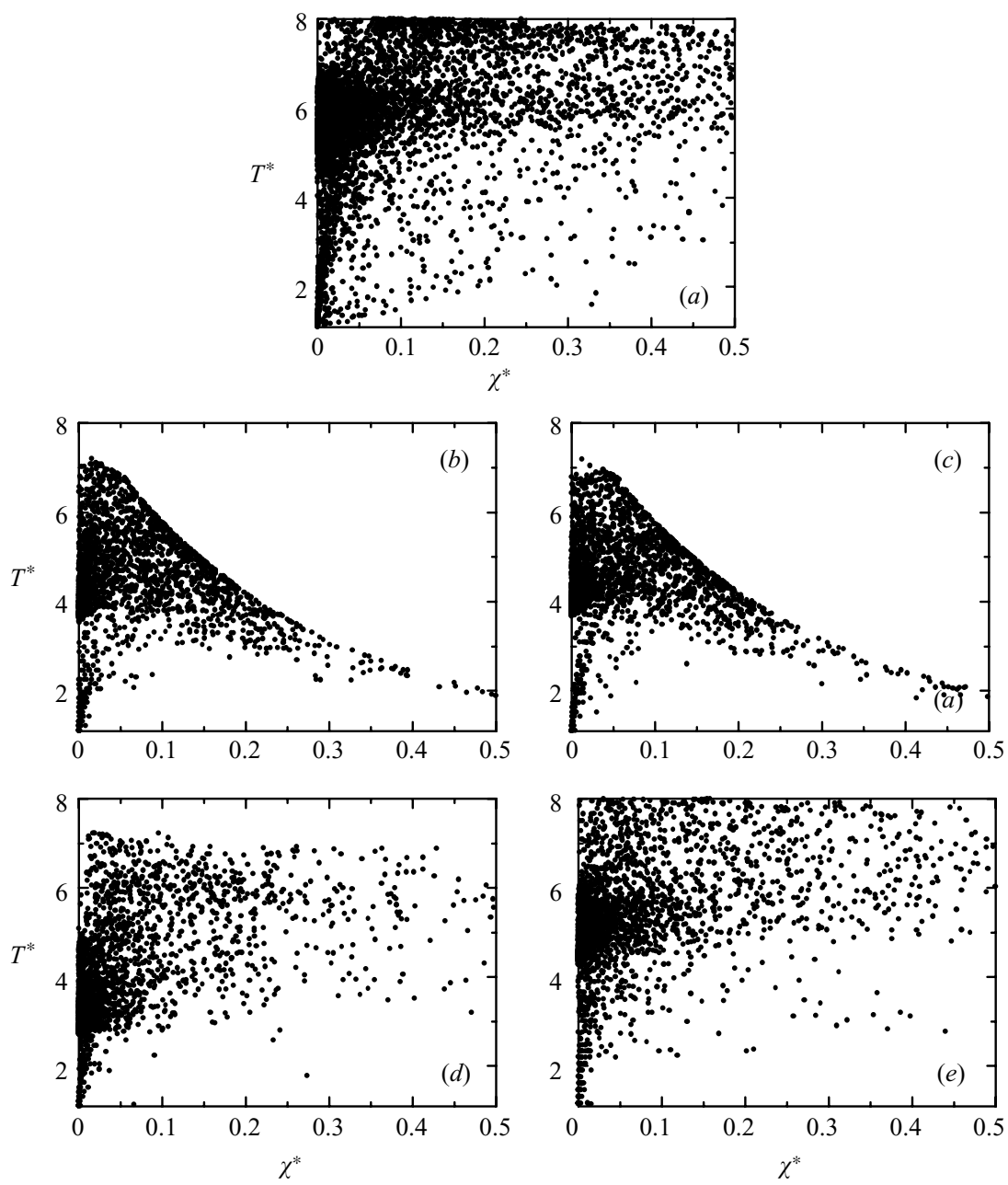

FIgURE 25. Comparison of scatter plots of instantaneous gaseous temperature $T^{*}$ against scalar dissipation rate $\chi^{*}$. (a) LAR, (b) LFM, (c) LFMM, (d) LFM-P and (e) LFM-E.

the product mass fraction as the progress variable does not function in this upstream region. On the other hand, LFM-E can predict the temperature difference between the fuel and oxidizer sides well. Although $\bar{Z}$ and $\overline{\chi^{*}}$ have the same value at $\bar{Z}=0.25$ on the fuel and oxidizer sides (see figure $26 a$ right), the $\overline{T^{*}}$ have different values, which clearly shows the superiority of FPVA-E. At the downstream location of $x^{*}=3.5$, no irregular behaviour is observed, but even LFM-E does not predict the $T^{*}-Z$ and $\chi^{*}-Z$ relations very well. This is because the high temperature originating from the premixed flames in the central region of the spray flame cannot be simulated either by SFM or FPVA.

\subsection{Energy transfer between the dispersed droplet and carrier gaseous phases}

From the above investigations, total enthalpy is found to be the preferred choice for use as the progress variable in FPVA, so understanding of the detailed behaviour of the total enthalpy within the spray flame is necessary. The variation of the spanwise profile of the time-averaged gaseous total enthalpy $\overline{h^{*}}$ is shown in figure 27 . Here, the values of $\overline{h^{*}}$ in the cases of LAR and LFM-E are obtained by directly solving the 

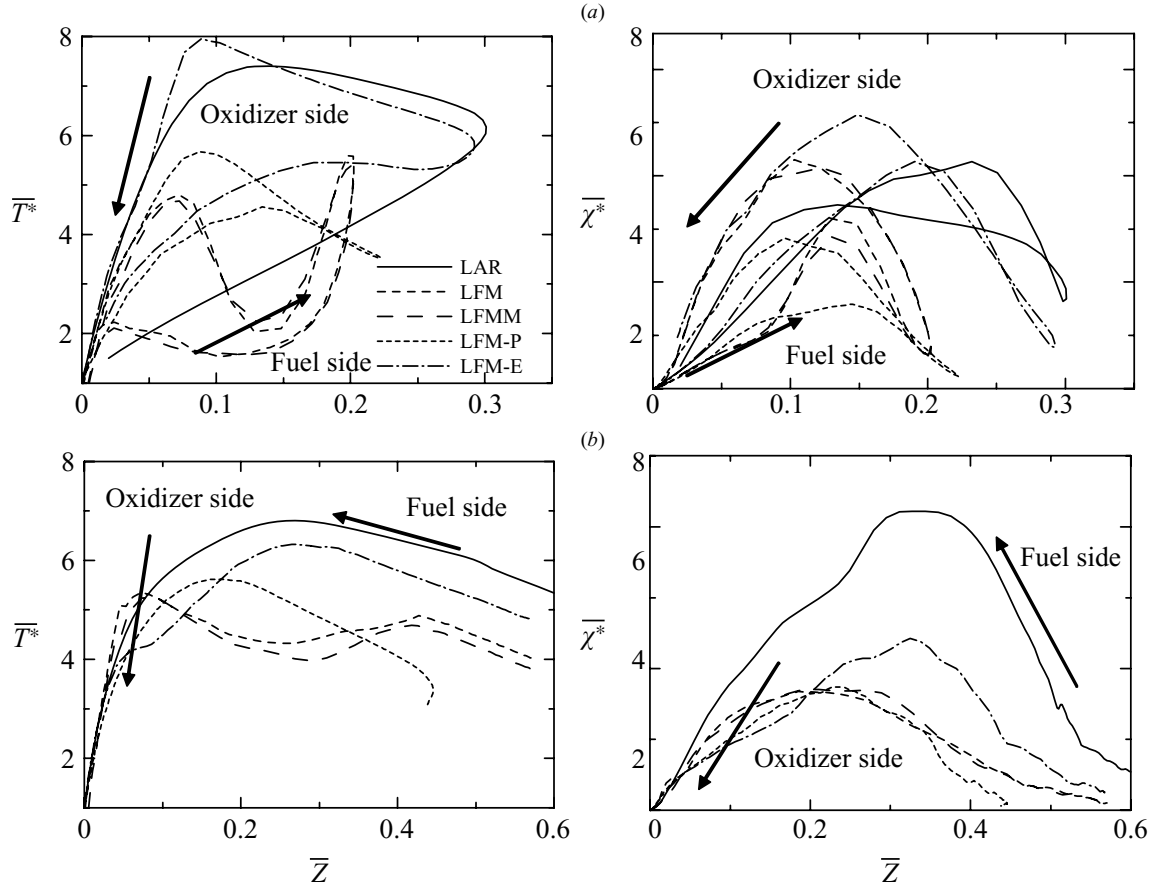

FIGURE 26. Relations between time-averaged gaseous temperature $\overline{T^{*}}$ and mixture fraction $\bar{Z}$ and between time-averaged scalar dissipation rate $\overline{\chi^{*}}$ and mixture fraction $\bar{Z}$ at two streamwise locations. (a) Upstream region $\left(x^{*}=0.5\right)$ and $(b)$ downstream region $\left(x^{*}=3.5\right)$.

conservation equation of $h$, whereas those in the other cases (i.e. LFM, LFMM and LFM-P) are obtained from the chemtable. Only LFM-E can properly capture the increase of LAR in the central region downstream, and the general trend. The increase in $\overline{h^{*}}$ is considered to result in energy transfer behaviour between the dispersed droplet and carrier gaseous phases.

Nakamura et al. (2005) revealed that in the phase-coupling source terms in the energy equation (2.8), the first term related to kinetic energy has less influence on the carrier gaseous phase than the second term related to energy transfer. The energy transfer term is divided into two terms as

$$
S C O=-\frac{1}{\Delta V} \sum_{N} c_{L} m_{d} \frac{\mathrm{d} T_{d}}{\mathrm{~d} t}, \quad S E V=-\frac{1}{\Delta V} \sum_{N} c_{L} T_{d} \frac{\mathrm{d} m_{d}}{\mathrm{~d} t},
$$

where $S C O$ and $S E V$ represent convective heat transfer and irreversible energy transfer (i.e. mass transfer) from the dispersed droplet phase to the carrier gaseous phase, respectively. The instantaneous distributions of $S C O, S E V$ and $S C O+S E V$ in the case of LAR are shown in figure 28. It is observed that $S C O$ shows a negative value in the upstream region, $S E V$ has a positive value in the whole region, and consequently, $S C O$ and $S E V$ are dominant in the upstream and downstream regions, respectively. This means that the gaseous total enthalpy is decreased in the upstream region and increased in the downstream region due to heat and mass transfer from the dispersed droplet phase, respectively. The reason why the effect of the heat transfer, which is the so-called droplet cooling effect, weakens in the downstream region is that the droplet temperature approaches the droplet boiling temperature downstream. This also means 

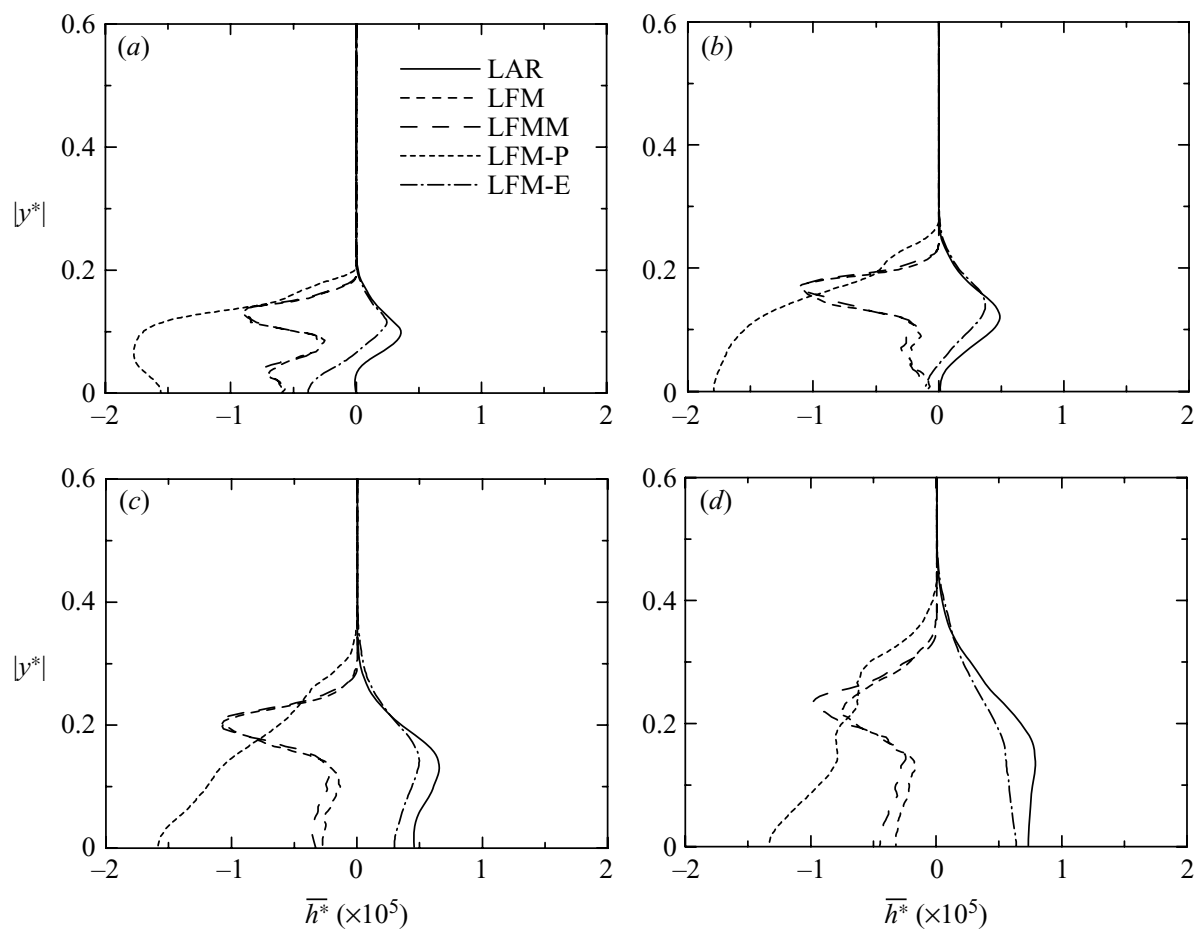

FIGURE 27. Comparison of spanwise profile of time-averaged gaseous total enthalpy $\overline{h^{*}}$ at four streamwise locations. (a) $x^{*}=1.0,(b) x^{*}=2.0$, (c) $x^{*}=3.0$ and $(d) x^{*}=4.0$.

(a)

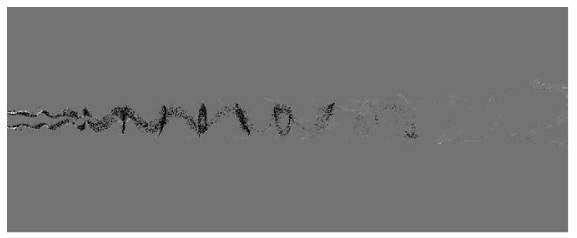

(b)

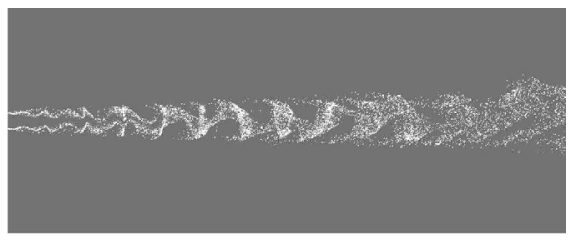

(c)

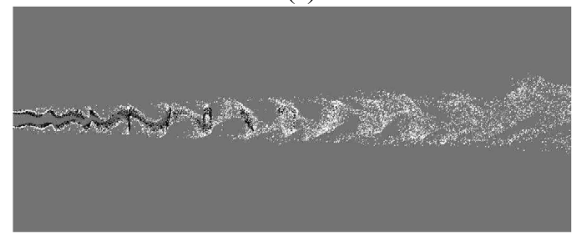

$-1.1 \times 10^{5}$ $+1.1 \times 10^{5}$

FIGURE 28. Instantaneous values of energy transfer terms between dispersed droplet and carrier gaseous phase of (a) SCO, (b) SEV and (c) SCO $+S E V$ in the case of LAR.

that the effect of the heat loss due to droplet evaporation weakens downstream. In figure 29, the quantitative variations of $S C O, S E V$ and $S C O+S E V$ are shown. The dominant contribution to the total energy transfer between the dispersed droplet and carrier gaseous phases shifts from heat transfer to mass transfer downstream. 

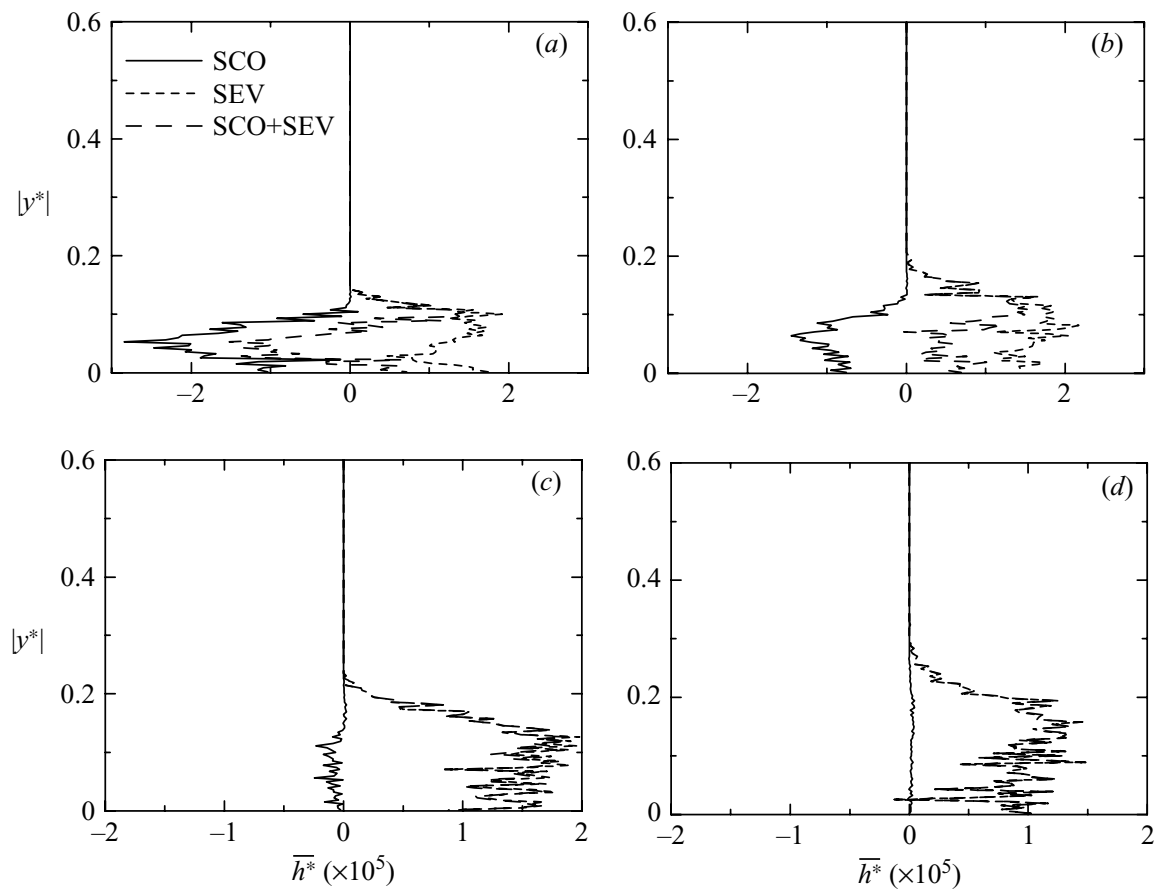

FIGURE 29. Spanwise profiles of time-averaged energy transfer terms of $S C O, S E V$ and $S C O+S E V$ at four streamwise locations in the case of LAR. (a) $x^{*}=1.0,(b) x^{*}=2.0$, (c) $x^{*}=3.0$ and $(d) x^{*}=4.0$.

In summary, the reason why total enthalpy is preferred for the progress variable in FPVA for spray combustion is that the behaviour of the gaseous total enthalpy is strongly affected by the energy transfer from the dispersed droplets, which cannot be accounted for either by SFM or FPVA-P. The energy transfer consisting of the heat and mass transfer can be predicted well by FPVA-E in combination with the enthalpy modification of the chemtable using (2.42).

\section{Conclusions}

The validity of the steady-flamelet model and flamelet/progress-variable approach for gaseous and spray combustion is investigated by performing two-dimensional direct numerical simulations (DNS) of gaseous and spray jet flames, and the detailed combustion characteristics are analysed in terms of combustion modelling. As a progress variable for the flamelet/progress-variable approach, product mass fraction has been employed in previous studies. In this study, on the other hand, a modified flamelet/progress-variable approach, in which total enthalpy is chosen as the progress variable, is proposed. The predicted combustion characteristics are compared with those obtained using an Arrhenius formation, in which a chemical reaction is directly solved in physical space. DNS coupled with this Arrhenius formation is assumed to reveal true flame behaviour.

The results from the DNS coupled with the Arrhenius formation show that the diffusion flame is dominant in the gaseous diffusion jet flame, whereas diffusion and premixed flames coexist in the spray jet flame. In other words, the spray jet flame is a partially premixed flame. The characteristics of the spray flame change from 
premixed-diffusion coexistent to diffusion-flame dominant downstream, and droplet group combustion appears in the diffusion-flame-dominant region. It is also found that although the flamelets on the unstable branch, which indicate low temperature under conditions of low mixture fraction and low scalar dissipation rate, are formed in the downstream region of the gaseous flame, they hardly appear in the spray combustion. This is because the mixture fraction of the spray flame tends to increase downstream.

Comparisons with the DNS results coupled with the steady-flamelet model and flamelet/progress-variable approach show that the flamelet/progress-variable approach, in which total enthalpy is employed as the progress variable, is superior to the other combustion models, and that this superiority is remarkable for the spray flame. This is because the behaviour of the gaseous total enthalpy is strongly affected by the energy transfer (i.e. heat transfer and mass transfer) from the dispersed droplet, and this effect can be accounted for only by solving the conservation equation of the total enthalpy.

It is also found that even using DNS with the flamelet/progress-variable approach employing the total enthalpy as the progress variable, the characteristics of the premixed flame appearing in the central region of the spray jet flame cannot be well predicted. The predicted gaseous temperature is underestimated in this region. This suggests that the combustion model for the partially premixed flame is necessary for the spray flame. In addition, the flamelets on the unstable branch are considered to appear in the region further downstream of the spray flame. The behaviour and the validity of the present flamelet/progress-variable approach in this region are still open to question.

The authors would like to thank Professor Fumiteru Akamatsu of Osaka University and Dr Hiroaki Watanabe of CRIEPI for their useful discussion. R.K. is grateful to Professor Heinz Pitsch and Dr Olivier Desjardins of the Center for Turbulence Research (CTR), Stanford University for their inspired discussion. Finally, the authors are grateful to Professor Youhei Morinishi of Nagoya Institute of Technology and the referees for their helpful advice regarding the numerical accuracy and the derivation of source terms of mixture fraction.

\section{REFERENCES}

Abramzon, B. \& Sirignano, W. A. 1989 Droplet vaporization model for spray combustion calculations. Intl J. Heat Mass Transfer 32, 1605-1618.

Apte, S. V., Gorokhovski, M. \& Moin, P. 2003 LES of atomizing spray with stochastic modeling of secondary breakup. Intl J. Multiphase Flow 29, 1503-1522.

Bellan, J. \& Summerfield, M. 1978 Theoretical examination of assumptions commonly used for the gas phase surrounding a burning droplet. Combust. Flame 33, 107-122.

Chiv, H. H., Kim, H. Y. \& Croke, E. J. 1982 Internal group combustion of liquid droplets. Proc. Combust. Inst. 19, 971-980.

Chiv, H. H. \& LiU, T. M. 1977 Group combustion of liquid droplets. Combust. Sci. Technol. 17, $127-131$.

Cook, A. W., Riley, J. J. \& KosÁLy, G. 1997 A laminar flamelet approach to subgrid-scale chemistry in turbulent flows. Combust. Flame 109, 332-341.

Domingo, P., Vervisch, L. \& Bray, K. 2002 Partially premixed flamelets in LES of nonpremixed turbulent combustion. Combust. Theory Modelling 6, 529-551.

Domingo, P., VervisCh, L. \& RÉVEILLON, J. 2005 DNS analysis of partially premixed combustion in spray and gaseous turbulent flame-bases stabilized in hot air. Combust. Flame 140, 172-195. 
Ham, F., Apte, S., Iaccarino, G., Wu, X., Herrmann, M., Constantinescu, G., Mahesh, K. \& MoIN, P. 2003 Unstructured LES of reacting multiphase flows in realistic gas turbine combustors. Annual Research Briefs, Center for Turbulence Research, NASA Ames/Stanford University, pp. 139-160.

Hollmann, C. \& Gutheil, E. 1998 Flamelet-modeling of turbulent spray diffusion flames based on a laminar spray flame library. Combust. Sci. Technol. 135, 175-192.

Jiménez, J., Liñán, A., Rogers, M. M. \& Higuera, F. J. 1997 A priori testing of subgrid models for chemically reacting non-premixed turbulent shear flows. J. Fluid Mech. 349, 149-171.

Kee, R. J., Dixon-Lewis, G., Warnaz, J., Coltrin, M. E. \& Miller, J. A. 1986 A fortran computer code package for evaluation of gas-phase multi-component transport properties. Sandia Report SAND86-8246.

KeE, R. J., Rupley, F. M. \& Miller, J. A. 1989 CHEMKIN-II: A FORTRAN chemical kinetics package for the analysis of gas phase chemical kinetics. Sandia Report SAND89-8009B.

Kim, J. \& Moin, P. 1985 Application of a fractional step method to incompressible Navier-Stokes equations. J. Comput. Phys. 59, 308-323.

Kurose, R., Desjardins, O., Nakamura, M., Akamatsu, F. \& Pitsch, H. 2004 Numerical simulations of spray flames. Annual Research Briefs, Center for Turbulence Research, NASA Ames/Stanford University, pp. 269-280.

Kurose, R. \& Komori, S. 1999 Drag and lift forces on a rotating sphere in a linear shear flow. J. Fluid Mech. 384, 183-206.

Kurose, R. \& Makino, H. 2003 Large eddy simulation of a solid-fuel jet flame. Combust. Flame 135, $1-16$.

Kurose, R., Makino, H., Komori, S., Nakamura, M., Akamatsu, F. \& Katsuki, M. 2003 Effects of outflow from the surface of a sphere on drag, shear lift, and scalar diffusion. Phys. Fluids 15, 2338-2351.

LEONARD, B. P. 1979 A stable and accurate convective modelling procedure based on quadratic upstream interpolation. Comput. Meth. Appl. Mech. Engng. 19, 59-98.

Miller, R. S. \& Bellan, J. 1999 Direct numerical simulation of a confined three-dimensional gas mixing layer with one evaporating hydrocarbon-droplet-laden stream. J. Fluid Mech. 384, 293-338.

Miller, R. S., Harstad, J. \& Bellan, J. 1998 Evaluation of equilibrium and non-equilibrium evaporation models for many-droplet gas-liquid flow simulations. Intl J. Multiphase Flow 24, 1025-1055.

Morinishi, Y., Lund, T. S., VAsilyev, O. V. \& Moin, P. 1998 Fully conservative higher order finite difference schemes for incompressible flow. J. Comput. Phys. 143, 90-124.

Nakamura, M., Akamatsu, F., Kurose, R. \& Katsuki, M. 2005 Combustion mechanism of liquid fuel spray in gaseous flame. Phys. Fluids 17, 123301.

Nicoud, F. 2000 Conservative high-order finite-difference schemes for low-Mach number flows. J. Comput. Phys. 158, 71-97.

Peters, N. 1984 Laminar diffusion flamelet models in non-premixed turbulent combustion. Prog. Energy Combust. Sci. 10, 319-339.

Peters, N. 2000 Turbulent Combustion. Cambridge University Press.

Pierce, C. D. 2001 Progress-variable approach for large-eddy simulation of turbulent combustion. Mech. Engng. Dept Rep. TF-80. Stanford University.

Pierce, C. D. \& Moin, P. 2004 Progress-variable approach for large-eddy simulation of non-premixed turbulent combustion. J. Fluid Mech. 504, 73-97.

Pitsch, H. 2000 Unsteady flamelet modeling of differential diffusion in turbulent jet diffusion flames. Combust. Flame 123, 358-374.

Pitsch, H. \& Duchamp DE Lageneste, L. 2002 Large-eddy simulation of premixed turbulent combustion using a level-set approach. Proc. Combust. Inst. 29, 2001-2008.

Pitsch, H. \& Peters, N. 1998 A consistent flamelet formulation for non-premixed combustion considering differential diffusion effects. Combust. Flame 114, 26-40.

Pitsch, H. \& Steiner, H. 2000 Large-eddy simulation of a turbulent piloted methane/air diffusion flame (Sandia flame D). Phys. Fluids 12, 2541-2554.

RÉveIllon, J. \& VERVISCH, L. 2005 Analysis of weakly turbulent dilute-spray flames and spray combustion regimes. J. Fluid Mech. 537, 317-347. 
Watanabe, H., Kurose, R., Hwang, S. \& Akamatsu, F. 2007 Characteristics of flamelet in spray flames formed in a laminar counterflow. Combust. Flame 148, 234-248.

Watanabe, H., Kurose, R., Komori, S. \& Pitsch, H. 2008 Effects of radiation on spray flame characteristics and soot formation. Combust. Flame 152, 2-13.

Westbrook, C. K. \& Dryer, F. L. 1984 Chemical kinetic modeling of hydrocarbon combustion. Prog. Energy Combust. Sci. 10, 1-57.

Yamashita, H., Shimada, M. \& Takeno, T. 1996 A numerical study on flamestability at the transition point of jet diffusion flames. Proc. Combust. Inst. 26, 27-34. 